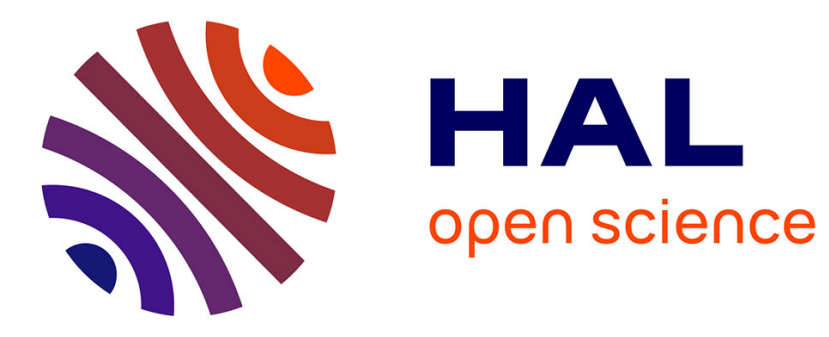

\title{
Elastostatic field distributions in polycrystals and cracked media
}

\author{
François Willot, Renald Brenner, Hervé Trumel
}

\section{To cite this version:}

François Willot, Renald Brenner, Hervé Trumel. Elastostatic field distributions in polycrystals and cracked media. Philosophical Magazine, 2020, 100 (6), pp.661-687. 10.1080/14786435.2019.1699669 . hal-02425491

\section{HAL Id: hal-02425491 \\ https://hal.science/hal-02425491}

Submitted on 17 Nov 2020

HAL is a multi-disciplinary open access archive for the deposit and dissemination of scientific research documents, whether they are published or not. The documents may come from teaching and research institutions in France or abroad, or from public or private research centers.
L'archive ouverte pluridisciplinaire HAL, est destinée au dépôt et à la diffusion de documents scientifiques de niveau recherche, publiés ou non, émanant des établissements d'enseignement et de recherche français ou étrangers, des laboratoires publics ou privés. 


\title{
ORIGINAL RESEARCH ARTICLE
}

\section{Elastostatic field distributions in polycrystals and cracked media}

\author{
F. Willot ${ }^{\mathrm{a}, \mathrm{b}^{*}}$, R. Brenner ${ }^{\mathrm{c}}$ and H. Trumel ${ }^{\mathrm{d}}$ \\ ${ }^{a}$ Mines ParisTech, PSL - Research University, Center for Mathematical Morphology, 35 rue $\mathrm{S}^{\mathrm{t}}$-Honoré, \\ 77300 Fontainebleau, France; ${ }^{\mathrm{b}}$ Mines ParisTech, PSL - Research University, Center for Materials, 63-65 \\ Rue Henri Auguste Desbruères, 91100 Corbeil-Essonnes, France; ' Sorbonne Université, CNRS, Institut \\ Jean Le Rond $\partial$ 'Alembert, 75005 Paris, France; ${ }^{\mathrm{d}}$ CEA, DAM, Le Ripault, 37260 Monts, France. \\ *Corresponding author. Email: francois.willot@ensmp.fr.
}

\author{
Reference \\ Philosophical Magazine (edited by Taylor \& Francis) 100(6), 661-687.
}

\begin{abstract}
This work addresses the problem of the reconstruction of the local fields distribution occurring in heterogeneous linear elastic solids. The constitutive heterogeneities are crystals and cracks. Through comparisons with FFT computations, it is shown that self-consistent estimates together with an assumption of normal distribution at the phase scale provide an accurate description of the elastostatic field histograms in polycrystals without cracks. In the case of inter and transgranular cracks, full-field FFT simulations indicate that the field histograms present van Hove singularities. Their natures are determined analytically in the low-density regime, in the case of an homogeneous medium containing cracks.
\end{abstract}

\section{KEYWORDS}

Polycrystals; Cracked media; Field fluctuation; Self-consistent Methods; Micromechanics; Van Hove singularities

\section{Introduction}

The probability distribution functions (or field histograms) of the local stress and strain fields in heterogeneous media are useful to gain insight on a material's mechanical response. On the one hand, distribution functions embody the material's macroscopic (homogenized) response, as well as statistics on the stress or strain extreme values (distribution tails). On the other hand, the information contained in probability distribution functions is highly condensed compared to the medium's full-field microscopic response. This property may be regarded as an advantage, since one-dimensional functions are easier to characterize and model than a material's full-field response, which requires an explicit description of the geometry.

Various works in micromechanics and in homogenization have investigated the distribution function of mechanical fields in heterogeneous media. Sevostianov [41] has modeled the distribution of the peak stresses occurring along the interface of porous inclusions in plane stress, using a Gumbel (double exponential) law. Idiart et al. [23] considered the fluctuations of mechanical fields in particulate viscoplastic composites, predicted by homogenization theories. Using FFT computations, they showed that the field distributions in the matrix are well approximated by Gaussian distributions in the linear case but strongly deviate from the latter when dealing with strong nonlinearities such as perfect plasticity. The distribution of elastic fields in polycrystals is considered in [26].

Probability distribution functions present, in general, Van Hove singularities, named after the work of Van Hove on the density of states of phonons in crystals [45]. Along Van Hove singularities, a distribution exhibits discontinuities, sharp peaks (blow-up and cusps) and nondifferentiable points. These singularities occur whenever the field's spatial gradient vanishes $[2$, 
34], typically extrema or saddle-points. Conversely, regions where the fields blow-up control the behavior of the distributions at infinity (distribution tails). Van Hove singularities occur in the probability distribution of the elastic fields in periodic media (see e.g. [50]), as well as in their electrical response [15]. Giordano [20] determined Van Hove singularities for the electric field surrounding an insulating crack. The elastic response is dealt with in [21]. The effect of a crack on the field distributions has also been considered in [16] for dielectric breakdown. The case of a random dispersion of inclusions has been investigated by Cule \& Torquato [15]. Comparing deterministic and random microstructures, the authors argue that, in random media, Van Hove singularities are smoothed out by disorder. Stokes flow in random porous media has also been investigated in [1].

The electrical (or dielectric) response has been considered in other works. Barthelemy and Orland [8] computed the local field distribution in random dielectric networks. The method makes use of the Lipmann-Schwinger equation and generalizes the classical self-consistent analytical treatment to field distributions. The method proposed by the authors show that the field distributions are well captured when taking into account first-neighbor interactions between bonds, and treating long-range interactions by an effective medium. Pellegrini [37] has modeled the field distributions in random dielectrics using Gaussian distributions to predict the response of "weakly nonlinear" media.

The present study aims at investigating up to which extent one may use homogenization theories to predict not only the mean (and second-order moments) of the fields but also the entire probability distribution functions of such fields, in linear elastic solids. The effect of a random dispersion of heterogeneities, made of crystals or cracks are examined. Self-consistent estimates and analytical results for the field surrounding an isolated crack are employed to model the elastic field distributions. Fourier-based computations are carried out to assess the validity of our approach, and to determine the presence of Van Hove singularities in random media. Furthermore, the asymptotics of the distribution tail are determined for certain stress components, in specific cases.

This article is organized as follows. The probability distribution functions of elastic fields occurring in 3D sound polycrystals are considered in Sec. (2). Sec. (3) deals with a homogeneous body containing an isolated crack, or a population of parallel, or randomly-oriented cracks, in plane strain, and investigates Van Hove singularities in the field distributions. Numerical and self-consistent results pertaining to the field histograms of cracked polycrystals in 3D are presented in Sec. (4).

\section{Field histograms in sound polycrystals}

\subsection{Multivariate distributions of the elastostatic fields}

A polycrystal made of elastically anisotropic grains can be considered as a composite material occupying a domain $\Omega$ whose constitutive phase $(r)$ represents a given crystalline orientation. Linear homogenization theories provide estimates for the intraphase first (i.e. mean) and secondorder moments $[12,27,38]$ of the strain field $\boldsymbol{\varepsilon}(\boldsymbol{x})$. For each phase $(r)$, they read

$$
\mathbf{M}_{\varepsilon}^{r}=\langle\varepsilon\rangle_{(r)} \quad \text { and } \quad \boldsymbol{\Sigma}_{\varepsilon}^{r}=\langle\varepsilon \otimes \varepsilon\rangle_{(r)}, \quad \forall r=1, \ldots, N .
$$

The multivariate probability distribution function of the tensorial field $\boldsymbol{\varepsilon}(\boldsymbol{x})$ in phase $(r)$ is defined as

$$
P_{\varepsilon}^{r}(\boldsymbol{t})=\lim _{\zeta \rightarrow 0} \frac{1}{\zeta} P\{\|\varepsilon-\boldsymbol{t}\|<\zeta\}=\frac{1}{\left|\Omega_{r}\right|} \int_{\Omega_{r}} \delta(\varepsilon(\boldsymbol{x})-\boldsymbol{t}) \mathrm{d} \boldsymbol{x}, \quad \boldsymbol{t} \in \mathcal{S}^{2},
$$

with $\delta$ the Dirac delta function, $\left|\Omega_{r}\right|$ the volume of phase $r$ and $\mathcal{S}^{2}$ the space of symmetric second-order tensors in $\mathbb{R}^{3}$ equipped with norm $\|\bullet\|=\sqrt{\bullet: \bullet}$. The intraphase first and second 
order moments of the strain field can thus be written

$$
\mathbf{M}_{\varepsilon}^{r}=\int_{\mathcal{S}^{2}} \boldsymbol{t} P_{\varepsilon}^{r}(\boldsymbol{t}) \mathrm{d} \boldsymbol{t} \quad \text { and } \quad \boldsymbol{\Sigma}_{\varepsilon}^{r}=\int_{\mathcal{S}^{2}}(\boldsymbol{t} \otimes \boldsymbol{t}) P_{\boldsymbol{\varepsilon}}^{r}(\boldsymbol{t}) \mathrm{d} \boldsymbol{t} .
$$

The overall distribution of the strain field $P_{\varepsilon}$ in domain $\Omega$ reads

$$
P_{\boldsymbol{\varepsilon}}(\boldsymbol{t})=\frac{1}{|\Omega|} \int_{\Omega} \delta(\varepsilon(\boldsymbol{x})-\boldsymbol{t}) \mathrm{d} \boldsymbol{x}=\sum_{r=1}^{N} f_{r} P_{\boldsymbol{\varepsilon}}^{r}(\boldsymbol{t}), \quad \boldsymbol{t} \in \mathcal{S}^{2},
$$

with $f_{r}=\left|\Omega_{r}\right| /|\Omega|$ the volume fraction of phase $(r)$. Similar definitions hold for the intraphase stress moments $\mathbf{M}_{\boldsymbol{\sigma}}^{r}$ and $\boldsymbol{\Sigma}_{\boldsymbol{\sigma}}^{r}$ as well as the stress probability distributions $P_{\boldsymbol{\sigma}}^{r}$ and $P_{\boldsymbol{\sigma}}$.

In order to use estimates on $\mathbf{M}_{\varepsilon}^{r}$ and $\boldsymbol{\Sigma}_{\varepsilon}^{r}$ provided by mean-field homogenization models, it is natural to consider multivariate normal (i.e. Gaussian) distributions for random tensor-valued variables. However, a symmetric second-order tensor in $\mathbb{R}^{3}$ can also be seen as a vector in $\mathbb{R}^{6}$. Therefore, the well-known theory of vector-valued normal distributions can advantageously be used $[4,9,25]$. The probability distribution function for the strain field $\boldsymbol{\varepsilon}(\boldsymbol{x})$ then reads [9]

$$
P_{\varepsilon}^{r}(\boldsymbol{t})=\frac{1}{\sqrt{\left|\mathbf{A}_{\varepsilon}^{r}\right|(2 \pi)^{6}}} \exp \left(-\frac{1}{2}\left(\boldsymbol{t}-\mathbf{M}_{\varepsilon}^{r}\right)^{T} \cdot\left(\mathbf{A}_{\varepsilon}^{r}\right)^{-1} \cdot\left(\boldsymbol{t}-\mathbf{M}_{\varepsilon}^{r}\right)\right), \quad \boldsymbol{t} \in \mathcal{V} .
$$

with $\mathcal{V}$ the vector space in $\mathbb{R}^{6}$. The covariance matrix $\mathbf{A}_{\varepsilon}^{r}$ is defined by

$$
\mathbf{A}_{\varepsilon}^{r}=\left\langle\left(\varepsilon-\mathbf{M}_{\varepsilon}^{r}\right) \otimes\left(\varepsilon-\mathbf{M}_{\varepsilon}^{r}\right)\right\rangle_{(r)}=\mathbf{\Sigma}_{\varepsilon}^{r}-\mathbf{M}_{\varepsilon}^{r} \otimes \mathbf{M}_{\varepsilon}^{r}
$$

and $\left|\mathbf{A}_{\varepsilon}^{r}\right|=\operatorname{det}\left(\mathbf{A}_{\varepsilon}^{r}\right)$. The inverse of the covariance matrix, which enters (5), is known as the precision matrix. Theorems available for vector-valued normal distributions [4] allow one to derive useful properties for the strain field distribution. In particular, any linear combination of the components of the strain field $\varepsilon(\boldsymbol{x})$ follows a univariate Gaussian intraphase distribution. Since the constitutive law is linear elastic, it follows that each component of the stress field $\boldsymbol{\sigma}(\boldsymbol{x})$ is also normally-distributed in phase $(r)$.

Let us diagonalize the covariance matrix $\mathbf{A}_{\varepsilon}^{r}$ as:

$$
\mathbf{A}_{\varepsilon}^{r}=\mathbf{B}_{\varepsilon}^{r} \cdot \mathbf{D}_{\varepsilon}^{r} \cdot\left(\mathbf{B}_{\varepsilon}^{r}\right)^{T}
$$

The orthogonal matrix $\mathbf{B}_{\varepsilon}^{r}$ is composed by the eigenvectors $\mathbf{b}_{i}^{r}(i=1, \ldots, 6)$ whereas the diagonal matrix $\mathbf{D}_{\varepsilon}^{r}$ give the eigenvalues $\lambda_{i}$ of the covariance matrix. Since the field $\varepsilon$ follows a normal intraphase distribution, the eigenvectors $\mathbf{b}_{i}^{r}$ are independent normal random fields. This result allows one to obtain statistical realizations of the intraphase strain field by generating vectors $\hat{\boldsymbol{y}}$ whose components $\hat{y}_{i}(i=1, \ldots, 6)$ are independent normal random variables with mean $\mu=0$ and variance $\sigma^{2}=1\left(\hat{y}_{i} \sim \mathcal{N}(0,1)\right)$. A particular realization $\widehat{\boldsymbol{\varepsilon}}^{r}$ of the strain field in phase $(r)$ reads (see e.g. [25, Chap. 4]):

$$
\widehat{\boldsymbol{\varepsilon}}^{r}=\mathbf{M}_{\varepsilon}^{r}+\mathbf{B}_{\varepsilon}^{r} \cdot \sqrt{\mathbf{D}_{\varepsilon}^{r}} \cdot \hat{\boldsymbol{y}} .
$$

Using the above, one may compute numerically the distribution of the equivalent von Mises strain $\varepsilon_{e q}=\sqrt{(2 / 3) \mathbf{e}: \mathbf{e}}$ with e the strain deviatoric tensor. More generally, Eqs. (7) and (8) allows one to compute the distribution of any function of the strain field components.

\subsection{Self-consistent estimates}

To get a statistical description of the local fields through mean-field homogenization, we adopt the self-consistent (SC) model which is well suited to describe the overall behavior of cell ma- 
terials [44] and polycrystalline media with moderate contrast [14, 29, 30]. It can be written [47]

$$
\left\langle\left\{[\mathbf{C}(\boldsymbol{x})-\widetilde{\mathbf{C}}]^{-1}+\mathbf{P}(\widetilde{\mathbf{C}})\right\}^{-1}\right\rangle=\mathbf{0}
$$

with $\mathbf{C}$ (resp. $\widetilde{\mathbf{C}}$ ) the local (resp. effective) elastic tensors and $\mathbf{P}$ the Hill microstructural tensor which depends on $\widetilde{\mathbf{C}}$ and the spatial distribution of the phases. The elastic properties being uniform per phase (i.e. crystalline orientation), that is:

$$
\mathbf{C}(\boldsymbol{x})=\sum_{r=1}^{N} \chi_{r}(\boldsymbol{x}) \mathbf{C}^{r}, \quad \forall \boldsymbol{x} \in \Omega,
$$

with $\chi_{r}(\mathbf{x})$ the characteristic function of phase $(r)$, relation (9) can be written

$$
\sum_{r=1}^{N} f_{r}\left[\left(\mathbf{C}^{r}-\widetilde{\mathbf{C}}\right)^{-1}+\mathbf{P}(\widetilde{\mathbf{C}})\right]^{-1}=\mathbf{0} .
$$

At the local scale, the self-consistent scheme provides information about the average fields for each crystalline orientation. For the strain field, for instance, the mean reads:

$$
\mathbf{M}_{\varepsilon}^{r}=\langle\varepsilon\rangle_{(r)}=\left(\mathbf{C}^{r}+\mathbf{C}^{*}\right)^{-1}:\left(\widetilde{\mathbf{C}}+\mathbf{C}^{*}\right): \bar{\varepsilon} .
$$

Besides, due to the quadratic dependence of the elastic energy on the strain field, the intraphase second moment of the strain field distribution can be obtained from the partial derivatives of the overall elastic energy $\widetilde{\omega}$ with respect to the local elastic tensors $\mathbf{C}^{r}[12,27,38]$

$$
\boldsymbol{\Sigma}_{\varepsilon}^{r}=\langle\varepsilon \otimes \varepsilon\rangle_{(r)}=\frac{2}{f_{r}} \frac{\partial \widetilde{\omega}}{\partial \mathbf{C}^{r}} \quad \text { with } \quad \widetilde{\omega}=\frac{1}{2} \bar{\varepsilon}: \widetilde{\mathbf{C}}: \overline{\boldsymbol{\varepsilon}} .
$$

In a general context of anisotropy, the SC estimate of the intraphase second-moment is solution of a linear system which requires the numerical integration of the Hill microstructural tensor $\mathbf{P}$ and its derivative $\partial \mathbf{P} / \partial \widetilde{\mathbf{C}}[13,14]$.

\subsection{FFT-based computations}

The distribution of the elastostatic fields in polycrystals can be addressed by considering unit-cell computations with different microstructure models. We consider the classical PoissonVoronoi tessellation [33, 35] and, for comparison, a modified Poisson-Johnson-Mehl partition of the 3D space, with anisotropic distance functions [17] (see Fig. 1a). The latter has been optimized to model a TATB (triamino-trinitrobenzene, a molecular triclinic crystal) polycrystal material [3] and in particular contains much more grains with elongated and non-convex shapes than in the Voronoi tessellation. The TATB crystals are strongly anisotropic. Their elastic behavior displays a general triclinic symmetry [10] which may be approached by transverse isotropy. In this study, we adopt the approximation of the crystal elastic tensor used in [19]. For simplicity, the crystallographic orientations in the grains are independent and uniformlydistributed on the sphere, leading to an isotropic effective response.

Numerical computations are carried out using a spectral Fourier method [36, 48] on voxel grids containing $1024^{3}$ voxels and about $10^{4}$ grains for the Voronoi model, and $256^{3}$ voxels and about 200 grains for the Johnson-Mehl model. As an example, we apply a macroscopic hydrostatic strain, normalized so that $\left\langle\varepsilon_{m}\right\rangle=1$. The field distributions $P_{\varepsilon_{x x}}$ are computed for three independent realizations of the Johnson-Mehl and Voronoi models (Fig. 1b). The 


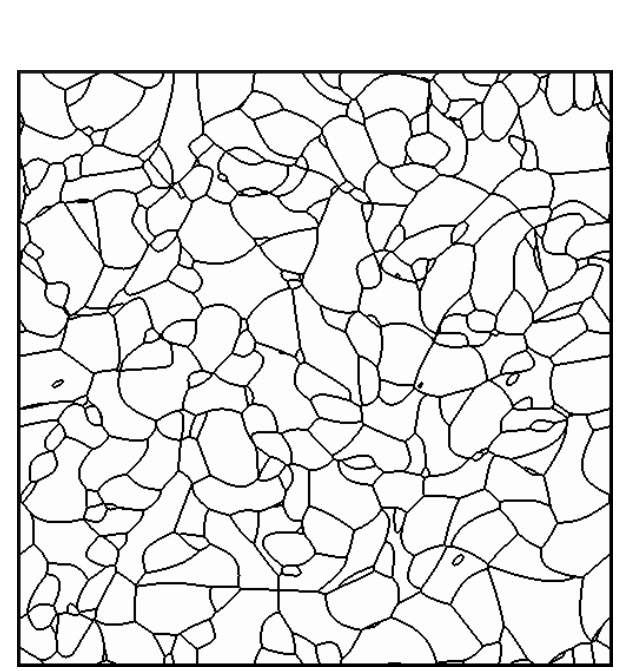

(a)

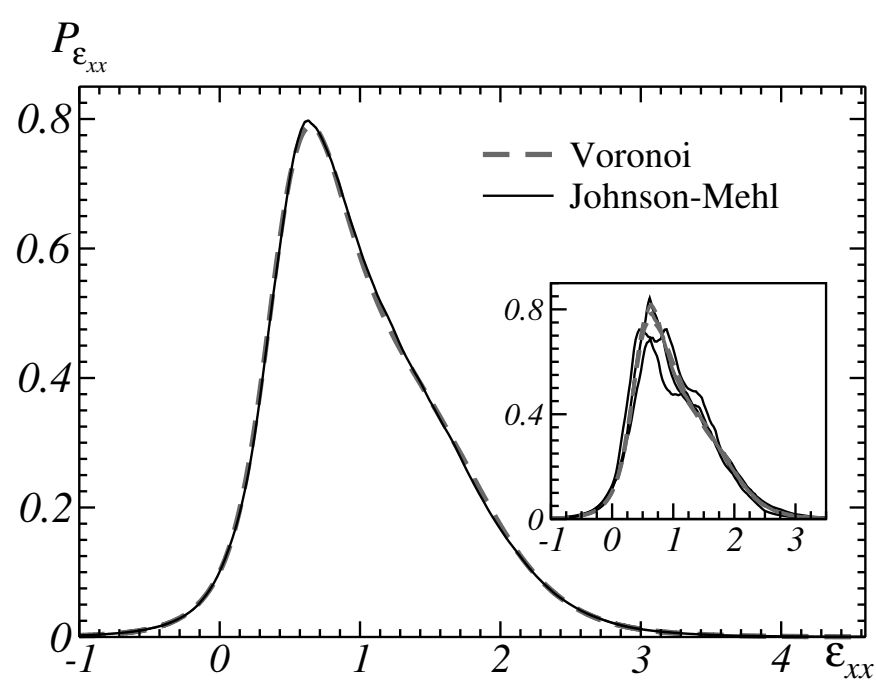

(b)

Figure 1. (a) Poisson-Johnson-Mehl tesselation of space made of elongated grains (2D cut). (b) Mean over three realizations of the distribution $P_{\varepsilon_{x x}}$ of the strain component $\varepsilon_{x x}$ in Poisson-Voronoi and Poisson-Johnson-Mehl polycrystals. Inset (bottomright): individual distributions, computed in each one of the three realizations.

overall distribution is nearly the same for all three realizations of the Voronoi model, except for small variations near the maximum $\varepsilon_{x x} \approx 0.6$ of the distribution (Fig. 1b, gray dashed line). As expected, the Johnson-Mehl model displays much higher variations with respect to the realizations (Fig. 1, inset). When averaged, however, the distributions for the Voronoi and Johnson-Mehl models nearly coincide (Fig. 1, solid and dashed lines). This result suggests that the histograms for the elastic fields are not very sensitive to the grains shape, at least for moderate anisotropy.

In the following, all computations are carried out using the Johnson-Mehl tessellation, discretized on a volume of $512^{3}$ voxels and containing about 1, 250 grains. In the sequel, the fullfield distributions are compared with the mean-field approximation based on the self-consistent model together with an assumption of intraphase normal distribution.

\subsection{Elastic fields distribution: self-consistent estimates vs. FFT results}

We evaluate the reconstruction of the fields distribution which is obtained by assuming a multivariate normal probability distribution within each phase $(r)(r=1, \ldots, N)$. Each distribution is characterized by the intraphase mean and variance which are estimated with the SC model. These "mean-field" distributions are compared with reference FFT results for isotropic TATB-like polycrystals made of grains with hexagonal symmetry (transversely isotropic elastic behavior). The implicit SC equation (11) is solved by considering an isotropic distribution of the phases (i.e. spherical inclusion for the ancillary Eshelby inclusion problem) and a set of crystalline orientations. To approximate an isotropic crystallographic texture (i.e. uniform orientation distribution function), the space of orientations has been discretized by considering a division of the standard triangle, for hexagonal crystal symmetry, in equal area domains [39]. The isotropic texture is represented by a set of 2160 crystalline orientations (i.e phases) with equal volume fractions. Our numerical results on the overall bulk and shear moduli agree with the analytical result of the SC estimate for isotropic polycrystals made of hexagonal grains [11].

The probability distribution functions $P_{\varepsilon}$ and $P_{\sigma}$, for field components and invariant quantities, have been computed for macroscopic hydrostatic $\left\langle\varepsilon_{m}\right\rangle=1$, shear $\left\langle\varepsilon_{x y}\right\rangle=0.5$ and uniaxial $\left\langle\varepsilon_{x x}\right\rangle=1$ strain loadings. The comparisons with the FFT computations show an overall good agreement with a correct description of the distributions asymmetry (Fig. 2). These results on linear elastic polycrystalline aggregates are consistent with those reported for linear viscous two-phase particulate composites [23]. 


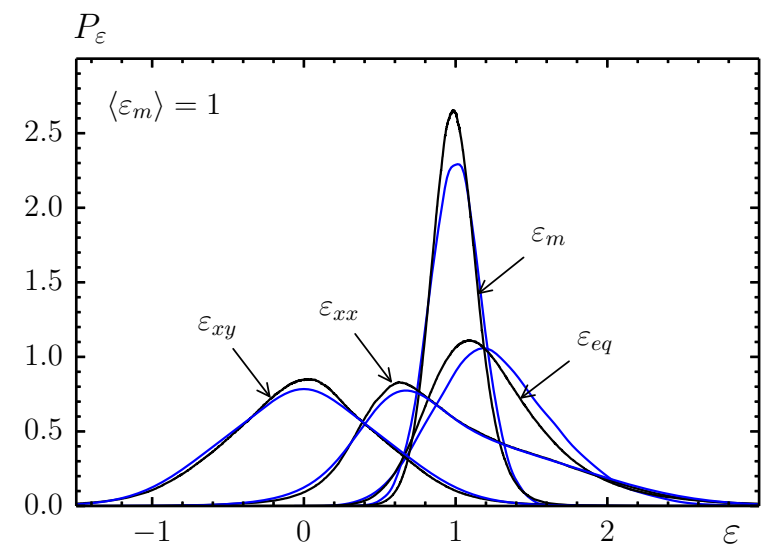

(a)

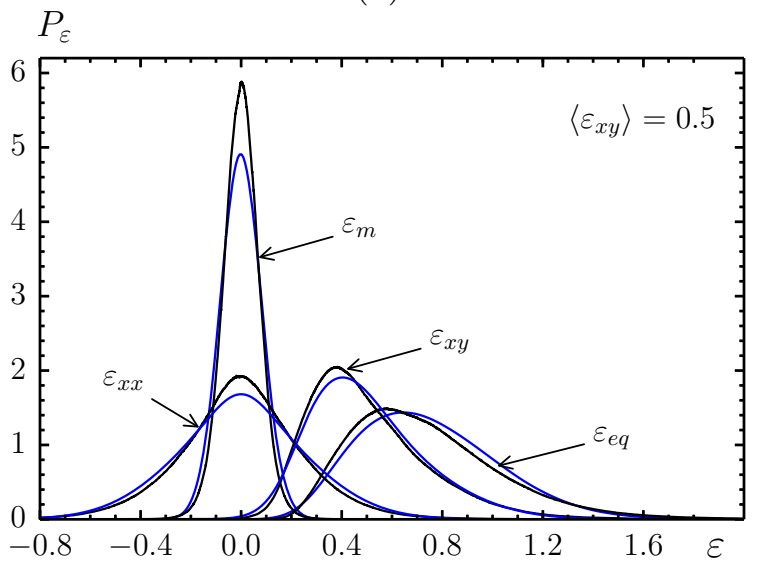

(c)

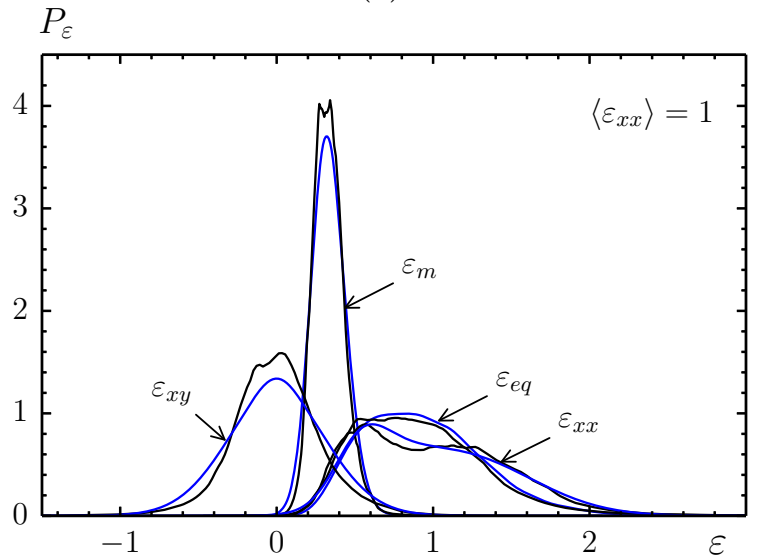

(e)

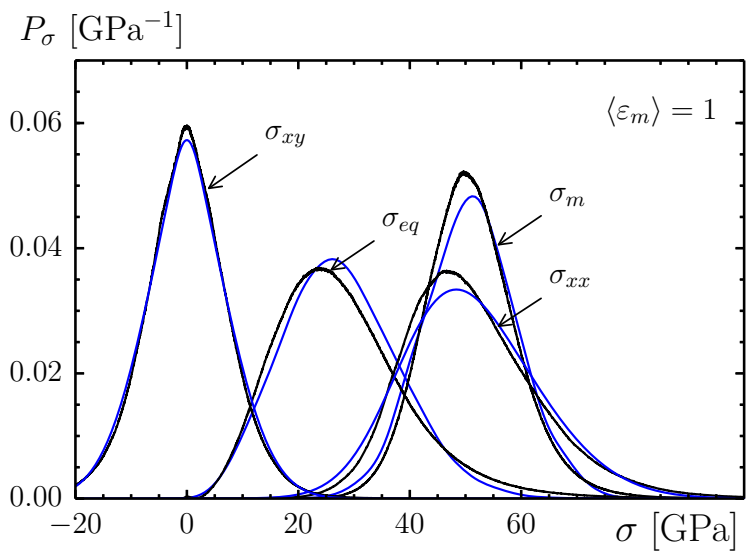

(b)

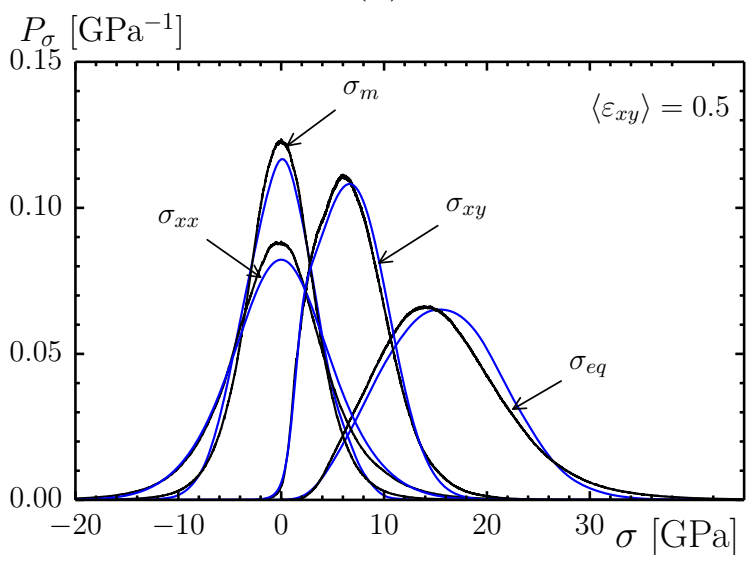

(d)

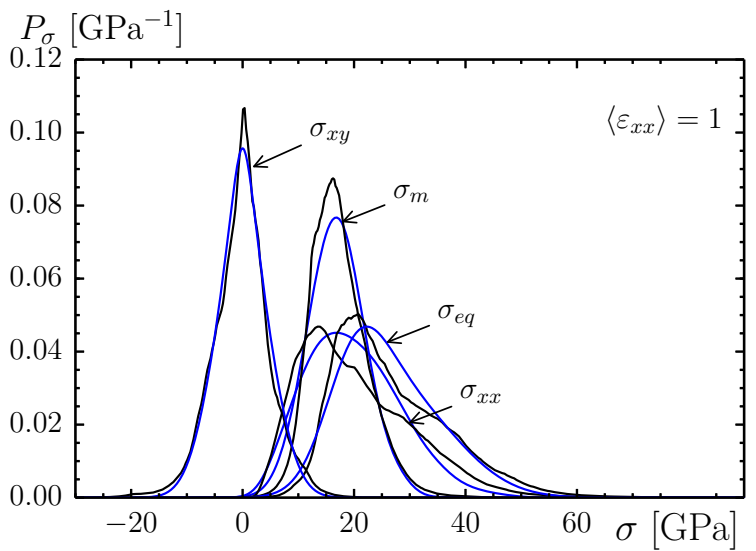

(f)

Figure 2. (a,c,e) Probability distribution function $P_{\varepsilon}$ for the mean and equivalent strains $\left(\varepsilon_{m}, \varepsilon_{e q}\right)$ and strain components $\left(\varepsilon_{x x}, \varepsilon_{x y}\right),(\mathrm{b}, \mathrm{d}, \mathrm{f})$ Probability distribution function $P_{\boldsymbol{\sigma}}$ for the mean and equivalent stress $\left(\sigma_{m}, \sigma_{e q}\right)$ and stress components $\left(\sigma_{x x}, \sigma_{x y}\right)$. Blue solid line (gray line in printed versions): Self-consistent estimate; Black solid line: FFT computation. (a,b) Overall hydrostatic loading with $\left\langle\varepsilon_{m}\right\rangle=1$. (c,d) Shear loading with $\left\langle\varepsilon_{x y}\right\rangle=0.5(\mathrm{e}, \mathrm{f})$ Uniaxial strain loading with $\left\langle\varepsilon_{x x}\right\rangle=1$.

\section{Field distributions in cracked media: homogeneous body under plane strain}

This section focuses on the elastic field distributions in a homogeneous body containing cracks and subjected to plane strain. In Subsection 3.1, a model problem made of an isolated crack or a population of randomly-oriented non-interacting cracks is investigated analytically. Emphasis is put on Van Hove singularities and a methodology is proposed to reconstruct the field distributions in the case of a population of interacting cracks. In subsection 3.2, the method's predictions are compared to FFT-based computations. 


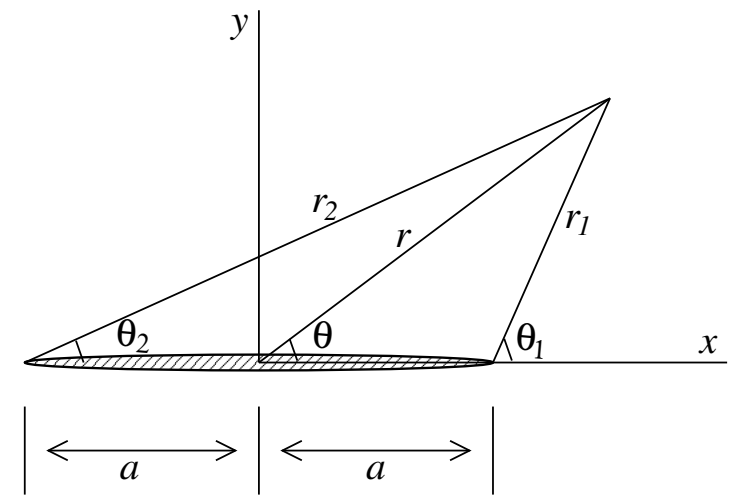

Figure 3. Coordinate systems around a crack (hashed area), in plane strain.

\subsection{Van Hove singularities}

As previously stated, Van Hove singularities are non-differentiable points in the probability distribution function $P(t)$ of a spatial field. These singularities are contributions of regions where the spatial gradient of the field vanishes. The type of singularity depends on the eigenvalues of the Hessian (second-order spatial derivatives) matrix being of the same or opposite signs [34]. For a field in 2D space, a saddle-point generates a logarithmic singularity of the type $P(t) \sim-\log \left|t-t_{0}\right|$ where $t_{0}$ is the value of the field at the saddle-point. In $2 \mathrm{D}$ again, a local extremum generates a finite jump in $P(t)$ at $t=t_{0}$, where $t_{0}$ is the field extremal value. In $3 \mathrm{D}$, saddle-points and local extrema induce powerlaw singularities with exponent $1 / 2$, therefore the probability distribution $P(t)$ remains finite while its derivative is unbounded. "Extended" Van Hove singularities [2] may in addition occur if the Hessian matrix has one or more zero eigenvalues. In 2D, blow-ups of the probability distribution function as a powerlaw of exponent $-1 / 2[2]$ or $-1 / 3[50]$ have been reported.

\subsubsection{Isolated crack}

We now examine histograms for the stress field surrounding an isolated crack. Vector and tensor components refer to a Cartesian coordinates system $\left(\mathrm{e}_{x}, \mathrm{e}_{y}, \mathrm{e}_{z}\right)$. The crack is modeled as an infinitely-thin cylinder of equation $|x|<a, y=0$, that is, its length is $2 a$ and its axis is parallel to $\mathrm{e}_{z}$ (see Fig. 3). The crack is subjected to plane strain in the $\left(\mathrm{e}_{x}, \mathrm{e}_{y}\right)$ plane (i.e. $\varepsilon_{i z} \equiv 0, i=x$, $y, z)$ and to remote biaxial stress loading, resulting in $\bar{\sigma}_{x x}=\bar{\sigma}_{y y}=\sigma_{k}>0, \bar{\sigma}_{x y}=0$ where $\overline{\boldsymbol{\sigma}}=\langle\boldsymbol{\sigma}\rangle_{\Omega}$ is the mean of the stress field over a very large domain $\Omega$ surrounding the crack.

In the vicinity of the crack tips, the stress components $\sigma_{i j}$ become singular [46]. In the three polar coordinate systems $(r, \theta),\left(r_{1}, \theta_{1}\right)$ and $\left(r_{2}, \theta_{2}\right)$ the stress around an isolated crack is expressed as [43]:

$$
\begin{aligned}
\sigma_{x y} & =\frac{\sigma_{k} r}{\sqrt{r_{1} r_{2}}}\left[\cos \left(\theta-\frac{\theta_{1}+\theta_{2}}{2}\right) \mp \frac{a^{2}}{r_{1} r_{2}} \sin \theta \sin \frac{3}{2}\left(\theta_{1}+\theta_{2}\right)\right], \\
\sigma_{x y} & =\frac{\sigma_{k} r a^{2}}{\left(r_{1} r_{2}\right)^{3 / 2}} \sin \theta \cos \frac{3}{2}\left(\theta_{1}+\theta_{2}\right) .
\end{aligned}
$$

Variables $r_{1,2}$ and $r$ denote the distances to the crack tips and to the mid-point along the crack, respectively, and $\theta_{1,2}, \theta$ are resp. the angles formed between the $x$-axis and the line joining the crack tips and the mid-point along the crack (see Fig. 3). The stress intensity factor "seen" by the crack is $\sigma_{k} / \sqrt{2 \pi a}$ [43]. Maps of the three stress components are shown in Figs. (4a-c).

Consider now the probability distribution $P_{i j}\left(\sigma_{k} ; t\right)$ of $\sigma_{i j}=t$, computed within a region $\Omega_{k}$. We assume that $\Omega_{k}$ is a circular domain with radius $D$ and surface fraction $f=\pi D^{2} / S$ and seek for the behavior of $P_{i j}\left(\sigma_{k} ; t\right)$ when $t=0$, or $t= \pm \infty$. Set $\theta_{1}=\pi-\mathrm{d} \theta_{1}$ and $\theta_{2}=\mathrm{d} \theta_{2}$ 
(a) $\sigma_{x x}[0 ; 4]$

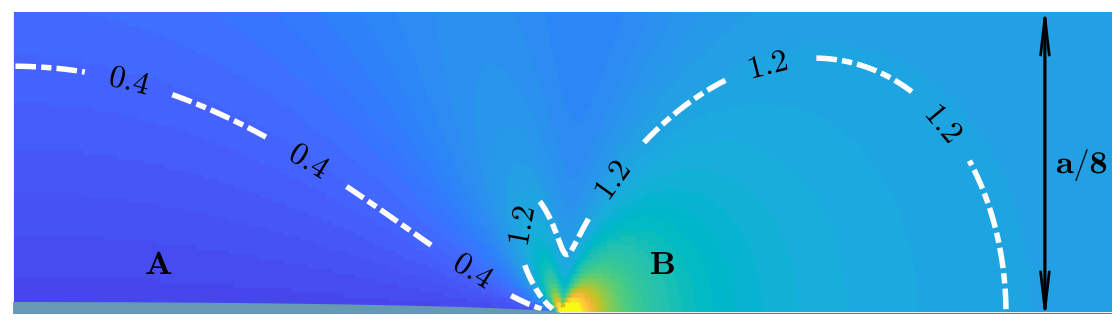

(b) $\sigma_{y y}[0 ; 4]$

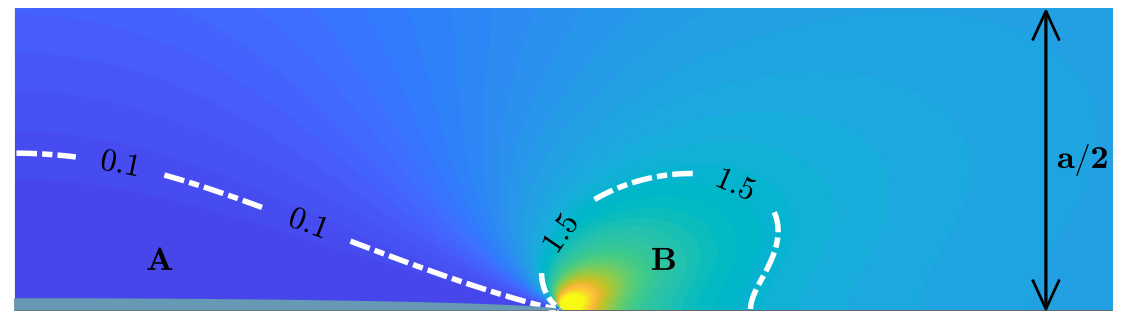

(c) $\sigma_{x y}[-2 ; 1]$

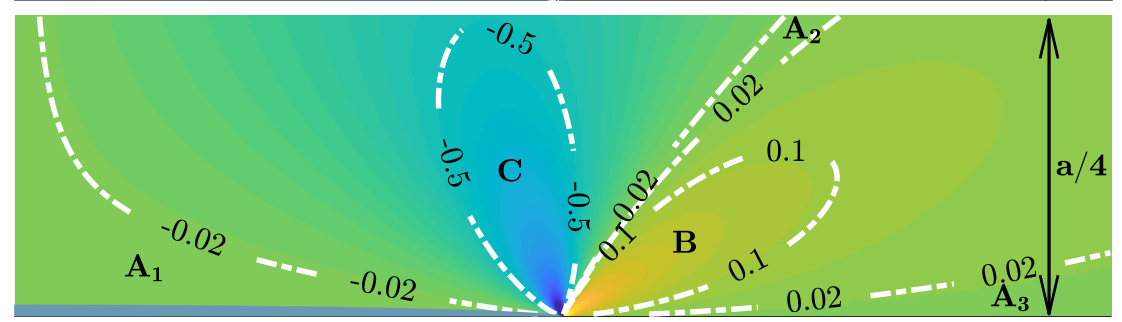

Figure 4. Maps of the stress components $\sigma_{i j}$ around an isolated crack with $\sigma_{k}=1 \mathrm{GPa}$ (Eqs. 14), in a domain $2 a>x>0$, $y>0$ (see Fig. 3). Annotated regions enclosed by contour lines determine the behavior of $P_{i j}(t)$ as $t \rightarrow 0, \pm \infty$. Regions $A, A_{1}$, $A_{2}, A_{3}: t \rightarrow 0$. Region $B: t \rightarrow \infty$. Region $C: t \rightarrow-\infty$. To highlight the field patterns, values lower than a minimum and higher than a maximum are thresholded out (see legend, units are GPa). Lowest values are shown in blue and highest values in yellow (dark and light gray resp. in printed version). Intermediate values in green and orange in the online version.

and expand (14) in the limit $\mathrm{d} \theta_{1} \rightarrow 0, \mathrm{~d} \theta_{2} \rightarrow 0$. One obtains:

$$
\sigma_{y y}=\sigma_{k} \frac{1+4(x / a)^{2}}{\left[1-(x / a)^{2}\right]^{7 / 2}}\left(\frac{y}{a}\right)^{3}+O\left(y^{5}\right) .
$$

When $t$ is small, the region where $0<\sigma_{y y}<t$ is accordingly delimited by the curves of equation:

$$
y= \pm a \frac{\left[1-(x / a)^{2}\right]^{7 / 6}}{\left[1+4(x / a)^{2}\right]^{1 / 3}} \frac{t^{1 / 3}}{\sigma_{k}^{1 / 3}}, \quad-a<x<a
$$

(region A, Fig. 4b). Integrating the above function leads to the extended Van Hove singularity:

$$
\begin{gathered}
P\left\{\sigma_{y y}(\boldsymbol{x})<t ; \boldsymbol{x} \in \Omega_{k}\right\}=\frac{4 a^{2} t^{1 / 3}}{\sigma_{k}^{1 / 3}\left|\Omega_{i}\right|} \int_{0}^{1} \frac{\mathrm{d} u\left(1-u^{2}\right)^{7 / 6}}{\left(1+4 u^{2}\right)^{1 / 3}}+O(t) \approx 0.701 \frac{a^{2} t^{1 / 3}}{D^{2} \sigma_{k}^{1 / 3}}, \\
P_{y y}\left(\sigma_{k} ; t\right) \approx 0.234 \frac{a^{2}}{D^{2} \sigma_{k}^{1 / 3} t^{2 / 3}} h(t), \quad t \rightarrow 0,
\end{gathered}
$$

where $h(\bullet)=\max (\operatorname{sign}(\bullet), 0)$ denotes the Heaviside function.

Component $\sigma_{x x}$ vanishes along the crack lips (region A, Fig. 4a), and its probability distri- 
bution function $P_{x x}$ presents a jump at $t=0$ :

$$
\sigma_{x x}=\frac{2 \sigma_{k}}{\left[1-(x / a)^{2}\right]^{3 / 2}} \frac{y}{a}+O\left(y^{2}\right), \quad P_{x x}\left(\sigma_{k} ; t\right)=\frac{3 a^{2}}{8 D^{2} \sigma_{k}} h(t), \quad t \rightarrow 0 .
$$

Component $\sigma_{x y}$ is zero along the two lines $y=0, x=0$ (regions $A_{1}$ and $A_{3}$, Fig. 4c) and along four curves starting from the crack tip at angles $\theta_{1}= \pm \pi / 3, \theta_{2}= \pm 2 \pi / 3$ (region $A_{2}$, Fig. $4 \mathrm{c}$ ). The gradient of the field $\sigma_{x y}$ is zero along the segment $y=0,-a<x<a$ (region $A_{1}$ ), and is non-zero in regions $A_{2}$ and $A_{3}$. The contributions to $P_{x y}(t)(t \ll 1)$ of regions $A_{2}$ and $A_{3}$ are accordingly finite, so that only the contribution of region $A_{1}$ matters. A Taylor expansion provides in the right-upper quadrant $x>0, y>0$ :

$$
\sigma_{x y}=-\frac{3 \sigma_{k} x / a(y / a)^{2}}{\left[1-(x / a)^{2}\right]^{5 / 2}}, \quad P_{x y}=-\frac{a^{2} \Gamma(-3 / 4) \Gamma(9 / 4)}{2 \sqrt{3} D^{2} \pi^{3 / 2} \sqrt{\sigma_{k}|t|}} \approx \frac{0.2839 a^{2}}{D^{2} \sqrt{\sigma_{k}|t|}}, \quad t \rightarrow 0,
$$

where $\Gamma(\bullet)$ is the extended factorial function, or Gamma function.

The tails of the distributions are now derived using the asymptotic near-tip expansions [43] (see regions $\mathrm{B}$ and $\mathrm{C}$ in Figs. 4a-c):

$$
\sigma_{\substack{x x \\ y y}}=\frac{\sigma_{k} \sqrt{a}}{\sqrt{2 r_{1}}} \cos \frac{\theta_{1}}{2}\left(1 \mp \sin \frac{\theta_{1}}{2} \sin \frac{3 \theta_{1}}{2}\right), \quad \sigma_{x y}=\frac{\sigma_{k} \sqrt{a}}{\sqrt{2 r_{1}}} \sin \frac{\theta_{1}}{2} \cos \frac{\theta_{1}}{2} \cos \frac{3 \theta_{1}}{2} .
$$

We solve the three equations $\sigma_{i j}=t$ for $t \rightarrow \pm \infty$ and obtain $r_{1}$ as a function of $\theta_{1}$ and $t$. The probability distributions $P_{x x}\left(\sigma_{k} ; t\right)$ and $P_{y y}\left(\sigma_{k} ; t\right)$ are obtained by integration of $-\left(4 / \pi D^{2}\right) r_{1} \partial_{t} r_{1}$ over $\theta_{1}$ in the range $[-\pi ; \pi]$. The distribution $P_{x y}\left(\sigma_{k} ; t\right)$ is derived by integration of the same quantity over the domain $[-\pi ;-\pi / 3] \cup[0 ; \pi / 3]$ (when $t \rightarrow \infty$, see region $\mathrm{B}$, Fig. 4c) and $[-\pi / 3 ; 0] \cup[\pi / 3 ; \pi]$ (when $t \rightarrow-\infty$, see region $\mathrm{C}$, Fig. 4c). This yields, to leading order-term in $t$ :

$$
\begin{aligned}
P_{x x}\left(\sigma_{k} ; t\right) & \sim \frac{177 a^{2} \sigma_{k}^{4}}{512 D^{2} t^{5}}, & t \rightarrow+\infty, \\
P_{y y}\left(\sigma_{k} ; t\right) & \sim \frac{1089 a^{2} \sigma_{k}^{4}}{512 D^{2} t^{5}}, & t \rightarrow+\infty, \\
P_{x y}\left(\sigma_{k} ; t\right) & \sim \frac{9 a^{2} \sigma_{k}^{4}}{1024 D^{2}|t|^{5}}, & t \rightarrow \pm \infty .
\end{aligned}
$$

The various probability distribution functions $\bar{P}_{i j}(t)=P_{i j}\left(\sigma_{k}=1 ; t\right)$, computed numerically from (14) on grids of $5000^{2}$ voxels, are shown in Fig. (5a). They present Van Hove singularities not only at $t=0, \pm \infty$ but also at various finite values of $t$. For reasons that will be clear later on, we do not investigate the later.

\subsubsection{Randomly-oriented non-interacting cracks}

Assume now that the material is made up of widely-separated cracks with uniformly-distributed orientations in the $\left(\mathrm{e}_{x}, \mathrm{e}_{y}\right)$ plane. Apply as previously a biaxial remote stress $\bar{\sigma}_{x x}=\bar{\sigma}_{y y}=\sigma_{k}>$ $0, \bar{\sigma}_{x y}=0$ in plane strain. Consider a crack oriented along a direction $0 \leq \beta \leq \pi$ with respect to the $x$-axis. The stress components $\sigma_{i j}^{\beta}$ are obtained by a rotation of angle $\beta$ in the plane $\left(\mathrm{e}_{x}\right.$, $\mathrm{e}_{y}$ ) of the stress tensor given in (14). For instance $\sigma_{x x}^{\beta}$ reads:

$$
\sigma_{x x}^{\beta}=\sigma_{x x} \cos ^{2} \beta+\sigma_{y y} \sin ^{2} \beta-\sigma_{x y} \sin (2 \beta) .
$$




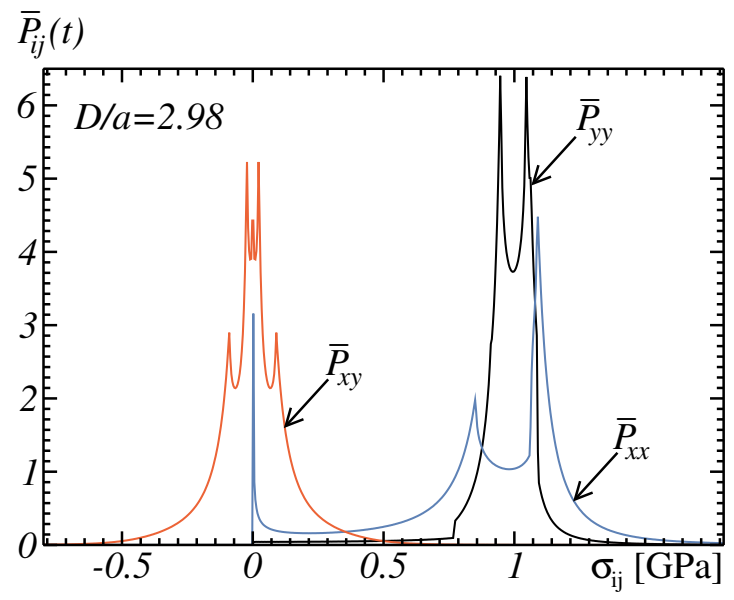

(a)

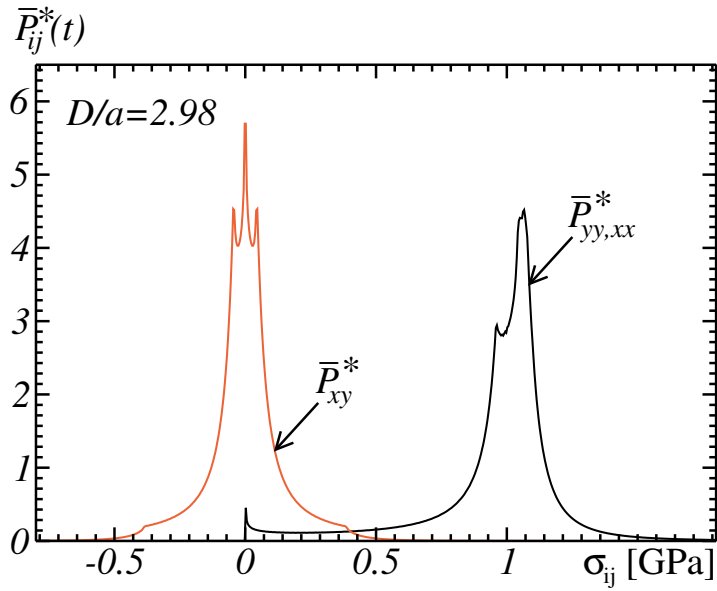

(b)

Figure 5. (a) Probability distribution functions $\bar{P}_{i j}(t)$ for the stress component $\sigma_{i j}$ around an isolated crack of length $a$, in a disk-shaped domain of radius $D$ centered around the crack. (b) Probability distribution functions $\bar{P}_{i j}^{*}(t)$ for the stress component $\sigma_{i j}$ around a set of isolated cracks of length $a$ with uniform orientations, in disk-shaped domains of radius $D$ centered around each crack.

The stress component $\sigma_{x x}^{\beta}$ vanishes along the crack tips and a Taylor expansion provides:

$$
\sigma_{x x}^{\beta}=2 \cos ^{2} \beta \sigma_{k}(y / a) /\left[1-(x / a)^{2}\right]^{3 / 2}, \quad y \rightarrow 0, \quad-a<x<a .
$$

Accordingly, the corresponding distribution presents a jump and:

$$
P_{x x}^{\beta}\left(\sigma_{k} ; t=0\right) \approx \frac{3 a^{2}}{8 D^{2} \sigma_{k} \cos ^{2} \beta} h(t), \quad t \rightarrow 0 .
$$

The above equation reduces to (18) in the special case $\beta=0$. The jump in (24) also blows up when $\beta=\pi / 2$, at which point $P_{x x}^{\beta}$ develops the singularity given in (17b).

Consider now the p.d.f. $P_{x x}^{*}\left(\sigma_{k} ; t\right)=\left\langle P_{x x}^{\beta}\left(\sigma_{k} ; t\right)\right\rangle_{\beta}$ of the stress fields surrounding cracks with stress factor $\sigma_{k}$, averaged over all orientations $\beta$. The smallest values of $\sigma_{k}$ are concentrated in a region near the crack tips. Furthermore, the size of this region is much more important for cracks oriented with an angle $\beta \approx \pi / 2$. Let us first examine the contribution of the values located along the line $x=0$. Set $r=y, \theta=\pi / 2, \theta_{1}+\theta_{2}=\pi, r_{1}=r_{2}$ in (14) and (22). We obtain:

$$
\sigma_{x x}^{\beta}(0, y)=\frac{\sigma_{k} y\left(a^{2} \cos (2 \beta)+a^{2}+y^{2}\right)}{\left(a^{2}+y^{2}\right)^{3 / 2}} .
$$

We solve $\sigma_{x x}^{\beta}=t$ for $\beta$ and determine the interval $\beta \in\left[\beta_{1}(y, t) ; \pi / 2\right]$ that contributes to values of $\sigma_{x x}^{\beta}$ in the range $[0 ; t]$. This interval is not empty whenever $y<y_{\max }=a t^{1 / 3} / \sqrt{\sigma_{k}^{2 / 3}-t^{2 / 3}}$. The region of interest, where the r.h.s. of (24) blows up, is $y \approx y_{\max }$ (as also confirmed by Eq. 16), therefore we make use of the variable change $y=y^{\prime} t^{1 / 3}$ in the following integral and obtain to leading-order in $t$ :

$$
\int_{0}^{y_{\max }(t)} \mathrm{d} y\left[\pi / 2-\beta_{1}(y, t)\right] \propto \frac{a t^{2 / 3}}{\sigma_{k}^{2 / 3}}, \quad t \rightarrow 0^{+} .
$$

Accordingly, the contribution of the line $x=0$ to $P_{x x}^{*}$ scales as $\sim t^{-1 / 3}$. The analytical computation of the contribution of the entire domain $\Omega$ proves cumbersome and we resort to numerical computations. The distribution $P_{x x}^{\beta}$ is computed by discretization of the field $\sigma_{x x}^{\beta}$ on a grid of 
$10,000^{2}$ pixels, and $P_{x x}^{*}$ is computed by averaging over 100 regularly-spaced values of the angle $\beta$. A fit of the numerical data, gives, with excellent agreement over two decades (see Fig. 6a):

$$
P_{x x}^{*}\left(\sigma_{k} ; t\right) \sim 0.44 \frac{a^{2} \sigma_{k}^{1 / 3}}{D^{2}} t^{-0.33}, \quad t \rightarrow 0^{+}
$$

which suggests an extended Van Hove singularity $P_{x x}^{*}=P_{y y}^{*} \sim t^{-1 / 3}$, as hinted by (26). This is a less singular behavior at the origin than that of the probability distribution $P_{y y}$ obtained for parallel cracks (Eq. 17b), which blows up as $\sim t^{-2 / 3}$.

Regarding component $\sigma_{x y}$, numerical computations also provide the following fit:

$$
P_{x y}^{*}\left(\sigma_{k} ; t\right) \approx-0.91 \frac{a^{2}}{D^{2}} \log \frac{|t|}{\sigma_{k}}, \quad t \rightarrow 0,
$$

with good agreement ranging over two and a half decades (see Fig. 6b). A powerlaw with small exponent $P_{x y}^{*} \sim t^{-0.1}$ instead of a logarithm can not be ruled out (Fig. 6b, inset) but appears less consistent with the data available. The logarithmic blow-up (28) is a weaker singularity than that obtained for the traction components $\sigma_{x x}$ or $\sigma_{y y}$ (Eq. 27).

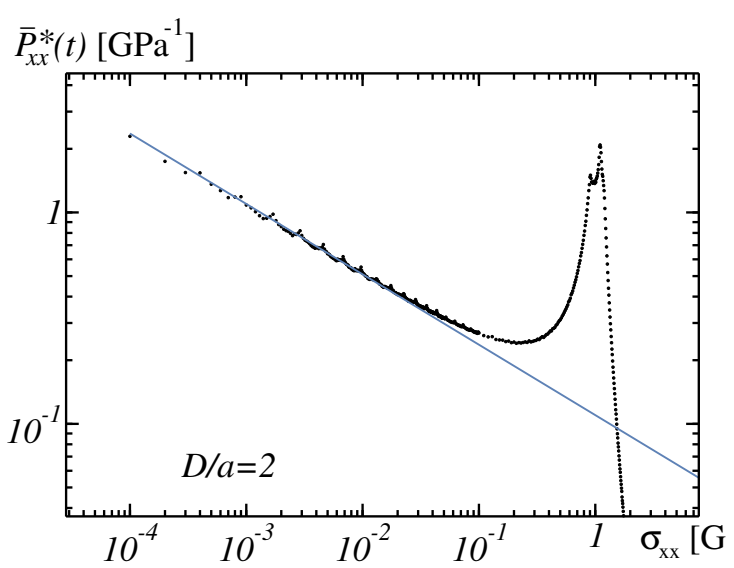

(a)

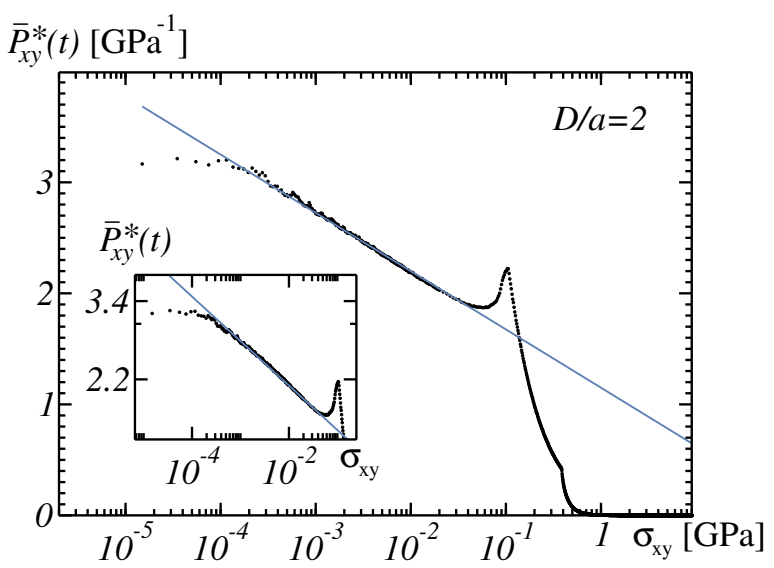

(b)

Figure 6. Numerical fit of the probability distribution functions $\bar{P}_{x x}^{*}$, in $\log$-log plot (a) and $\bar{P}_{x y}^{*}$, in semi-log plot (b) for components $\sigma_{x x}$ and $\sigma_{x y}$, in media with randomly-oriented cracks. Solid line in (a): fit (27); in (b): fit (28), up to a constant term. Inset in (b): log-log plot of the data with fit $\bar{P}_{x y}^{*}=5.5(a / D)^{2}\left(t / \sigma_{k}\right)^{-0.1}$.

Finally, the behavior at $t= \pm \infty$ is computed analytically in the same manner as in Sec. 3.1.1. We obtain, the $x$ - and $y$-axis being statistically equivalent directions:

$$
\begin{aligned}
P_{y y}^{*}\left(\sigma_{k} ; t\right) & \sim \frac{585 a^{2} \sigma_{k}^{4}}{512 D^{2} t^{5}}, \quad t \rightarrow+\infty \\
P_{x y}^{*}\left(\sigma_{k} ; t\right) & \sim \frac{9 a^{2} \sigma_{k}^{4}}{1024 D^{2}|t|^{5}}, \quad t \rightarrow \pm \infty .
\end{aligned}
$$

Observe that the prefactor for $P_{x x}^{*}\left(\right.$ or $P_{y y}^{*}$ ) lies in-between that of $P_{x x}$ and $P_{y y}$, while it is unchanged for the shear component $x y$ (Eq. 21c).

The probability distribution functions $\bar{P}_{i j}^{*}(t)$, are shown in Fig. (5b). They are computed numerically from (14) and (22) on grids of $5000^{2}$ voxels. The parameter $\beta$ is discretized along 200 values. Note that, as for the distribution functions $\bar{P}_{i j}$, they present Van Hove singularities at finite values of $t$, not investigated in the present work. 


\subsubsection{Parallel and randomly-oriented interacting cracks}

Consider now a population of interacting identical cracks, either parallel to one another or randomly-oriented. To take into account crack interactions, we assume that the overall distribution of the stress field around the cracks is the same as that of a set of isolated cracks subjected to random stress intensity factors, with a probability law for the stress intensity factors to be determined. As in the previous sections, the material is subjected to plane strain and in-plane biaxial stress loading. The cracks density is monitored by the non-dimensional parameter $\eta=N_{c} / S a^{2}$ where $S=|\Omega|$ is the surface area of the domain $\Omega$ and $N_{c}$ the number of cracks within the domain.

Consider a particular component $\sigma_{i j}$ in a domain $\Omega_{k}$ containing a crack with remote stress $\sigma_{k}$, and with field distribution $P_{i j}$. Clearly:

$$
P_{i j}\left(\sigma_{k} ; t\right)=\frac{1}{\left|\sigma_{k}\right|} \bar{P}_{i j}\left(\frac{t}{\sigma_{k}}\right),
$$

where $\bar{P}_{i j}(t)=P_{i j}(1 ; t)$ is the distribution obtained when taking $\sigma_{k}=1$. For a continuous distribution $q(s)$ of stress intensity factors in domains $\Omega_{k}\left(k=1, \ldots, N_{c}\right)$ with $N_{c}$ very large, the probability distribution of $\sigma_{i j}$ over $\Omega$, is given by the convolution product:

$$
\widetilde{P}_{i j}(t)=\int_{-\infty}^{+\infty} \mathrm{d} s \frac{q(s)}{|s|} \bar{P}_{i j}\left(\frac{t}{s}\right)=\int_{-\infty}^{+\infty} \mathrm{d} u \frac{\bar{P}_{i j}(u)}{|u|} q\left(\frac{t}{u}\right), \quad \int_{-\infty}^{\infty} \mathrm{d} s q(s)=1 .
$$

We also denote $\widetilde{P}_{i j}^{*}(t)$ the orientation-averaged distribution $\widetilde{P}_{i j}(t)$, equal to:

$$
\widetilde{P}_{i j}^{*}(t)=\int_{-\infty}^{+\infty} \mathrm{d} s \frac{q^{*}(s)}{|s|} \bar{P}_{i j}^{*}\left(\frac{t}{s}\right), \quad \int_{-\infty}^{\infty} \mathrm{d} s q^{*}(s)=1,
$$

where $\bar{P}_{i j}^{*}(t)=P_{i j}^{*}\left(\sigma_{k}=1 ; t\right)$. The fields moments $(n=1,2, \ldots)$ read:

$$
\left\langle t^{n}\right\rangle_{\widetilde{P}_{i j}}=\left\langle t^{n}\right\rangle_{\bar{P}_{i j}}\left\langle t^{n}\right\rangle_{q}, \quad\left\langle t^{n}\right\rangle_{\widetilde{P}_{i j}^{*}}=\left\langle t^{n}\right\rangle_{\bar{P}_{i j}^{*}}\left\langle t^{n}\right\rangle_{q^{*}}
$$

In principle, these formula can be used to reconstruct $q$ knowing $\widetilde{P}_{i j}$ and $\bar{P}_{i j}$, under some conditions [42]. In practice, the problem is ill-posed and efficient numerical algorithms must be implemented [24]. In the present situation, our knowledge of the two moments in (33) leads to the natural assumption that $q$ and $q^{*}$ are Gaussian kernels:

$$
q(s)=\frac{1}{\sqrt{2 \pi v_{q}}} \exp \left(-\frac{\left(s-\mu_{q}\right)^{2}}{2 v_{q}}\right), \quad q^{*}(s)=\frac{1}{\sqrt{2 \pi v_{q}^{*}}} \exp \left(-\frac{\left(s-\mu_{q}^{*}\right)^{2}}{2 v_{q}^{*}}\right),
$$

where we assume $\mu_{q}>0, \mu_{q}^{*}>0$. In doing so, we smooth out singularities that may be present in distributions $\bar{P}_{i j}$ and $\bar{P}_{i j}^{*}$ except possibly at $t=0$ and $t=+\infty$, which are fixed under the transformation $t \mapsto t / s$. We emphasize that other choices are possible. Kushch et al. [28], notably, modeled the distribution of stress intensity factors in similar heterogeneous media using a Gumbel (double exponential) law.

Let us examine the possible Van Hove singularities of $\widetilde{P}_{i j}$ and $\widetilde{P}_{i j}^{*}$ as $t \rightarrow \pm \infty$. Using Eqs. (21) 
we obtain:

$$
\begin{aligned}
\widetilde{P}_{y x}(t) & \sim p_{y x}|t|^{-5} \int_{0}^{\infty} \mathrm{d} s s^{4} q(\operatorname{sign}(t) s), \quad t \rightarrow \pm \infty \\
\widetilde{P}_{x y}(t) & \sim p_{x y}|t|^{-5} \int_{0}^{\infty} \mathrm{d} s s^{4}[q(s)+q(-s)], \quad t \rightarrow \pm \infty \\
p_{x x} & =\frac{177 a^{2}}{512 D^{2}}, \quad p_{y y}=\frac{1089 a^{2}}{512 D^{2}}, \quad p_{x y}=\frac{9 a^{2}}{1024 D^{2}},
\end{aligned}
$$

where $p_{i j} t^{-5}$ is the asymptotic behavior of $\bar{P}_{i j}(t)$ as $t \rightarrow \infty$ provided by Eqs. (21). The same relations are obtained for $\widetilde{P}_{i j}^{*}$ with prefactors $p_{i j}$ given by $(29 \mathrm{a})$ instead. Note that $\widetilde{P}_{x x}, \widetilde{P}_{y y}$, $\widetilde{P}_{x x}^{*}$ and $\widetilde{P}_{y y}^{*}$ possess distribution tails as $t \rightarrow-\infty$, unlike distributions $\bar{P}_{x x}$ and $\bar{P}_{y y}$. The tails are asymmetric: the prefactor at $t=-\infty$ is lower than that obtained in the $t=+\infty$ limit.

The behavior of $\widetilde{P}_{y y}$ as $t \rightarrow 0$ is determined as:

$$
\widetilde{P}_{y y}(t) \sim p_{y y}^{\prime}|t|^{-\nu} \int_{0}^{\infty} \mathrm{d} s s^{\nu-1} q(\operatorname{sign}(t) s), \quad t \rightarrow 0^{ \pm},
$$

where $p_{y y}^{\prime} t^{-\nu}(\nu=2 / 3)$ is the asymptotic behavior of $\bar{P}_{y y}$ as $t \rightarrow 0^{+}$(see Eq. 17b). The behavior of $\widetilde{P}_{x x}^{*}=\widetilde{P}_{y y}^{*}$ as $t \rightarrow 0$ is obtained by taking $\nu=0.33$ and by replacing prefactor $p_{y y}^{\prime}$ by that provided by Eq. (27). The behavior of $\widetilde{P}_{x y}$ as $t \rightarrow 0$ is obtained as:

$$
\widetilde{P}_{x y}(t) \sim p_{x y}^{\prime}|t|^{-1 / 2} \int_{0}^{\infty} \mathrm{d} s s^{-1 / 2}[q(s)+q(-s)], \quad t \rightarrow 0 .
$$

where $p_{x y}^{\prime} t^{-1 / 2}$ is the asymptotic behavior of $\bar{P}_{x y}$ given in (19).

We finally consider distribution $\widetilde{P}_{x x}$ which takes a finite value when $t=0^{+}$and is zero when $t<0$ (18). The integral in (32) is splitted into two intervals $\left[0 ; t / t_{0}\right]$ and $\left[t / t_{0} ; \infty\right]$. Assuming $0<t \ll t_{0} \ll 1$ :

$$
\begin{aligned}
& \int_{0}^{t / t_{0}} \frac{\mathrm{d} s}{s} q\left(\frac{t}{s}\right) \bar{P}_{x x}(s) \approx-q\left(t_{0}\right) \bar{P}_{x x}(0) \log \left(t_{0}\right), \\
& \int_{t / t_{0}}^{\infty} \frac{\mathrm{d} s}{s} q\left(\frac{t}{s}\right) \bar{P}_{x x}(s) \approx-q\left(t_{0}\right) \bar{P}_{x x}(0) \log \frac{t}{t_{0}} .
\end{aligned}
$$

A similar behavior is obtained when $t \rightarrow 0^{-}$so that:

$$
\widetilde{P}_{x x}(t) \sim-q(0) \bar{P}_{x x}\left(0^{+}\right) \log |t|, \quad t \rightarrow 0^{ \pm} .
$$

This singularity, a logarithmic blow-up, is generated by regions around cracks subjected to nearly zero remote stress, consistently with (32). Notice that $\bar{P}_{x x}$ displays a finite jump at $t=0$ (Eq. 18), unlike $\widetilde{P}_{x x}$. A similar treatment, carried out on distribution $\bar{P}_{x y}^{*}$ (Eq. 28), leads to:

$$
\widetilde{P}_{x y}^{*}(t) \sim \frac{q(0)}{2} \log ^{2}|t|, \quad t \rightarrow 0^{ \pm}
$$

Again, the nature of the singularity is different from the logarithmic blow-up found for $\bar{P}_{x y}^{*}$ in (28).

The Van Hove singularities at $t=0$ and $t= \pm \infty$ of the distribution functions $\bar{P}_{i j}, \widetilde{P}_{i j}, \bar{P}_{i j}^{*}$ and $\widetilde{P}_{i j}^{*}$ are summarized in Tab. (1). As previously noted, the singularities of $\widetilde{P}_{i j}$ are different 


\begin{tabular}{|c|c|c|c|c|c|}
\hline & $\sigma_{i j}$ & $\bar{P}_{i j}$ & $\widetilde{P}_{i j}$ & $\bar{P}_{i j}^{*}$ & $\widetilde{P}_{i j}^{*}$ \\
\hline \multirow{3}{*}{$t=0^{ \pm}$} & $\sigma_{x x}$ & $h(t)$ & $-\log |t|$ & $h(t)|t|^{-0.33}$ & $h_{\bullet}(t)|t|^{-0.33}$ \\
\cline { 2 - 6 } & $\sigma_{y y}$ & $h(t)|t|^{-2 / 3}$ & $h \bullet(t)|t|^{-2 / 3}$ & & \\
\cline { 2 - 6 } & $\sigma_{x y}$ & $|t|^{-1 / 2}$ & $|t|^{-1 / 2}$ & $-\log |t|$ & $\log ^{2}|t|$ \\
\hline \multirow{2}{*}{$t= \pm \infty$} & $\sigma_{x x}, \sigma_{y y}$ & $h(t)|t|^{-5}$ & $h_{\bullet}(t)|t|^{-5}$ & $h(t)|t|^{-5}$ & $h_{\bullet}(t)|t|^{-5}$ \\
\cline { 2 - 6 } & $\sigma_{x y}$ & \multicolumn{4}{|c|}{$|t|^{-5}$} \\
\hline
\end{tabular}

Table 1. Van Hove singularities of the probability distribution functions of the stress components $\sigma_{i j}$ in the non-interacting model of parallel $\left(\bar{P}_{i j}\right)$ or randomly-oriented $\left(\bar{P}_{i j}^{*}\right)$ cracks, and in the respective interacting models $\left(\widetilde{P}_{i j}\right.$ and $\left.\widetilde{P}_{i j}^{*}\right)$. Constant prefactors (that do not depend of $t$ ) are omitted for clarity. The term $h_{\bullet}(t)$ designates a function with a finite jump at $t=0$, of the type $b+b^{\prime} h(t)$ with $b, b^{\prime}>0$.

from that of $\bar{P}_{i j}$. For instance $\widetilde{P}_{y y}$ develops powerlaw singularities when $t \rightarrow 0^{-}$or $t \rightarrow-\infty$, contrarily to $\bar{P}_{y y}$ which has support on $\left[0 ; \infty\left[\right.\right.$. Likewise, $\widetilde{P}_{x x}$ develops a logarithmic singularity when $t \rightarrow 0^{-}$, contrarily to $\bar{P}_{x x}$. Also, as will be seen later on, distributions $\widetilde{P}_{i j}(t)$ and $\widetilde{P}_{i j}^{*}(t)$ do not present singularities at finite (non-zero) values of $t$.

\subsection{FFT-based computations}

\subsubsection{Reconstruction of the overall stress distribution vs. FFT data}

We compute numerically the stress distributions occurring in the solid phase of a $2 \mathrm{D}$ Boolean set [40] of cracks subjected to plane strain. The cracks are parallel to one another. Again, we use the FFT scheme with "backward-forward" finite difference [49], appropriate to the presence of discretized cracks [18], and computations are carried out on two independent realizations of the Boolean model, discretized on a $2048^{2}$-pixels grid. We follow [18] and discretize the cracks as thin rectangles of width $w=1.5$ voxel. In all computations hereafter, the overall applied stress is by convention equal to $1 \mathrm{GPa}$ so that $\left\langle\sigma_{x x}\right\rangle=\left\langle\sigma_{y y}\right\rangle=1$. The cracks have a finite thickness, therefore the mean of the stress field in the solid phase is slightly above $1 \mathrm{GPa}$.

The probability distribution function $\widetilde{P}_{x x}(t)$ is compared with FFT results in Fig. (7a). The first and second moments of the distribution of the stress intensity factor $q$, used to compute $\widetilde{P}_{x x}(t)$, are adjusted so that the moments of $\widetilde{P}_{x x}(t)$ fit the corresponding FFT data (see 33). The values of $a$ and $D$ are chosen so that the crack density parameter $\eta$ is the same in the FFT computations and in each domain $\Omega_{k}$, i.e.

$$
\eta=\frac{N_{c} a^{2}}{S}=\frac{a^{2}}{\pi D^{2}}
$$

where $N_{c}=60$ is the number of cracks in FFT computations, $S=2048^{2}$ is the surface of the unit cell, $a=25$ pixels, and so $\eta \approx 0.035, D \approx 75$ pixels. It is emphasized that FFT numerical results have been used, in the present work, to tailor the first and second-order moments of $\widetilde{P}_{x x}$. In principle, however, such moments could be determined using self-consistent estimates. For a cracked body in plane strain, these estimates, which include the case of an anisotropic embedding medium, have been derived analytically by Nemat-Nasser \& Horri [22].

A similar procedure is used in the random case. Consider the low crack-density regime $\eta \ll 1$. Eq. (33) gives the first and second-moments of $q^{*}$, given that of $\widetilde{P}_{i j}^{*}$. The latter are provided by FFT computations, carried out on a $8192^{2}$ pixels grid. We use $a=25$ pixels, $N_{c}=20, D=129$ and $\eta=0.011$. The mean and second-order moments of $\widetilde{P}_{y y}^{*}$, given by FFT computations, are 1.0007 and 1.0279 resp. and the mean and variances of $q$ are 1.0103 and $310^{-4}$ resp. Results are shown in Fig. (7b).

The method however breaks down when $\eta$ is larger than about 0.05 , at which point (33) predicts a negative variance for $q$, so that this constraint can not be satisfied anymore. Indeed, 


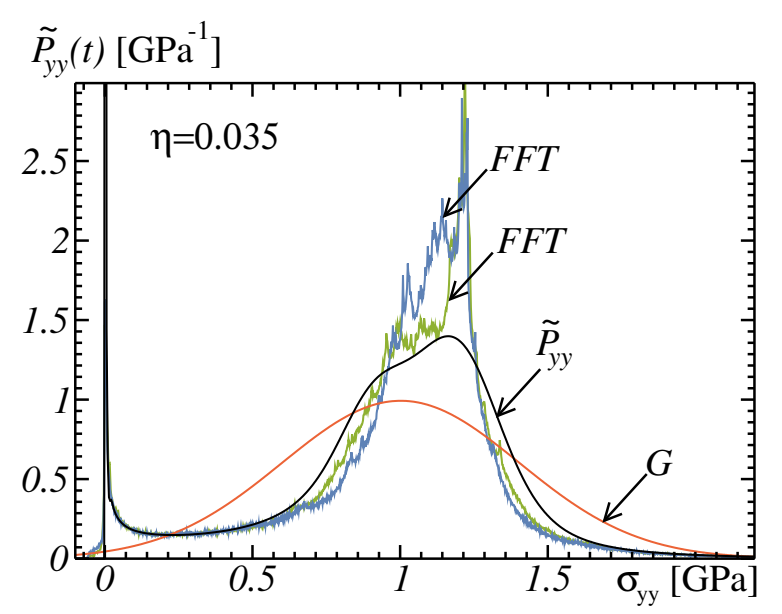

(a)

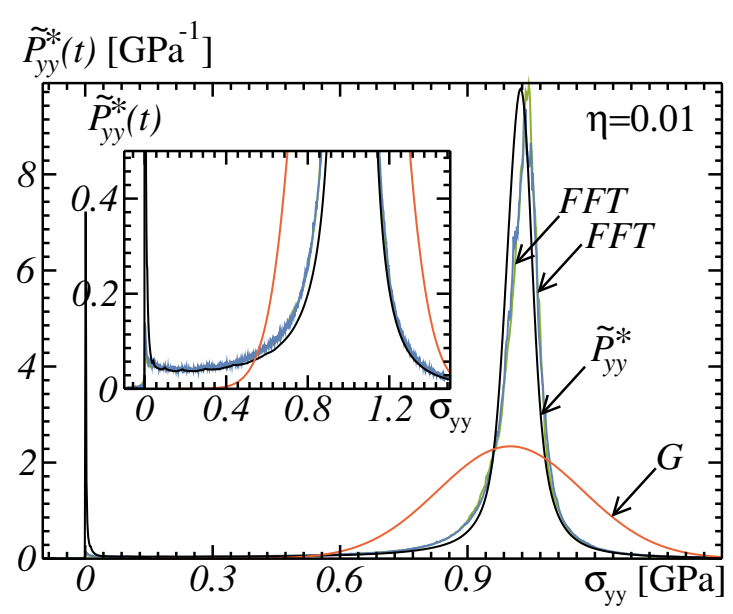

(b)

Figure 7. Probability distribution functions $\widetilde{P}_{y y}(t)$ and $\widetilde{P}_{y y}^{*}(t)$ (a and b, resp., solid black lines) for the stress component $\sigma_{y y}$, in a matrix containing a set of (a) parallel and (b) randomly-oriented cracks, in plane strain. Comparison with FFT results (online: solid blue and green lines, printed version: gray lines) obtained on two random realizations, and to a Gaussian distribution (solid line marked G). Fig. (b), inset: enlargement in the region $\widetilde{P}_{y y}^{*}(t)<0.5$.

our method assumes that all cracks are loaded in mode-I. In random media, in reality, the regions in which cracks are embedded are subjected to varying loadings, combining several modes. Furthermore, in the present method, the distribution $q$ entering (33) does not depend on the stress component considered, hence, one can not simultaneously satisfy all set of constraints on the first and second moments of the stress components. Taking into account other modes would, in theory, provide additional degrees of freedom, at the expense of a more analyticallyinvolved treatment.

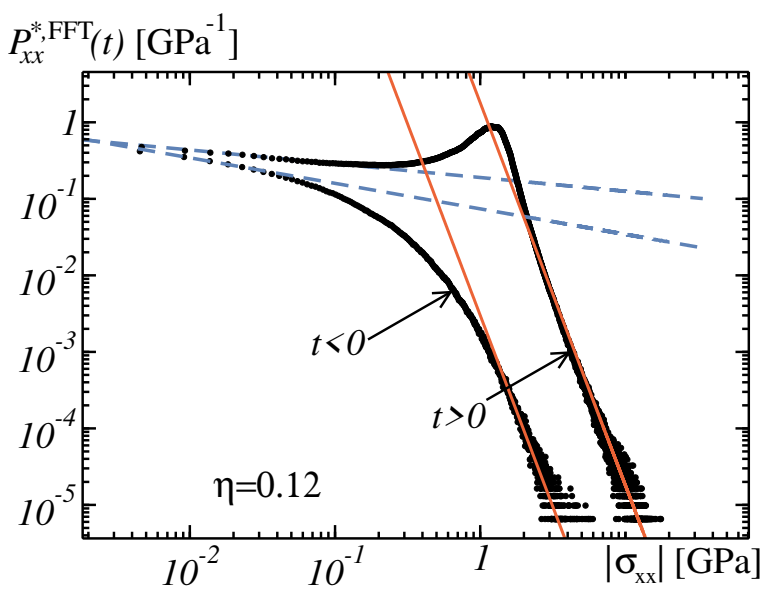

(a)

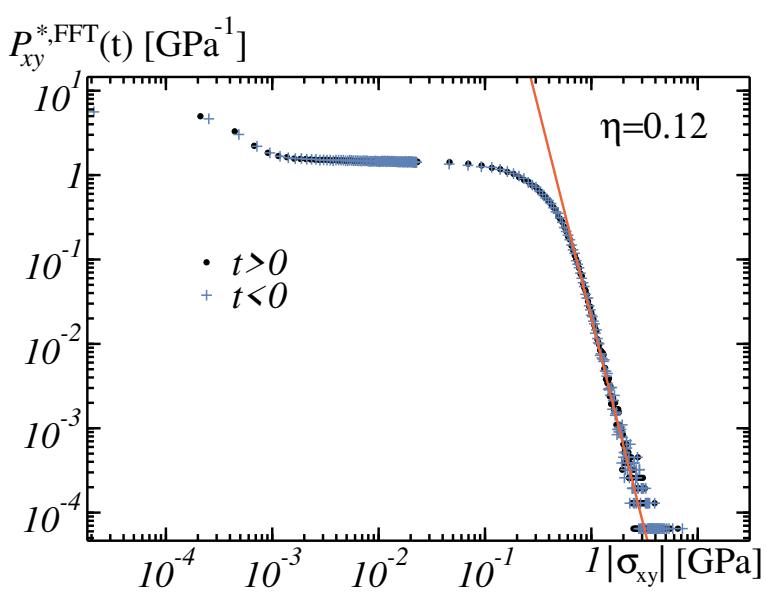

(b)

Figure 8. Probability distribution functions $P_{y y}^{*, F F T}$ and $P_{x y}^{*, F F T}$ for the stress components $\sigma_{y y}(\mathrm{a})$ and $\sigma_{x y}(\mathrm{~b})$ in randomlyoriented cracks, in log-log plot, as predicted by FFT computations. Solid and dotted lines: powerlaw fit (42) and (43) in the domains $|t| \ll 1$ and $t \gg 1$.

\subsubsection{Van Hove singularities}

We now focus on the singularities of the probability distribution functions $P_{i j}^{*, F F T}$, predicted by numerical FFT computations. All computations are carried out on two independent realizations of a Boolean model of cracks, discretized on a $8192^{2}$ pixel grid containing $N_{c}=200$ cracks. To highlight possible Van Hove singularities, we choose a crack density equal to $\eta=0.12$ with 
$a=25$ pixels. The following powerlaw behavior is observed. When $t \rightarrow \pm \infty$ :

$$
\begin{aligned}
& P_{x x, y y}^{*, F F T} \approx|t|^{-5} \times\left\{\begin{aligned}
1.8, & t \rightarrow+\infty, \\
0.03, & t \rightarrow-\infty,
\end{aligned}\right. \\
& P_{x y}^{*, F F T} \approx 0.02|t|^{-5}, \quad t \rightarrow \pm \infty
\end{aligned}
$$

(see Figs. 8a-b). When $t \rightarrow 0$ :

$$
P_{x x, y y}^{*, F F T} \approx\left\{\begin{array}{cc}
0.19 t^{-0.18}, & t \rightarrow 0^{+} \\
0.074|t|^{-0.33}, & t \rightarrow 0^{-}
\end{array}\right.
$$

Fits (43) should be taken with care as the two powerlaw regimes are observed on two decades only, or less (see Fig. 8a). The behavior of $P_{x y}^{*, F F T}$ as $t \rightarrow 0$ is not provided here, as FFT results are inconclusive.

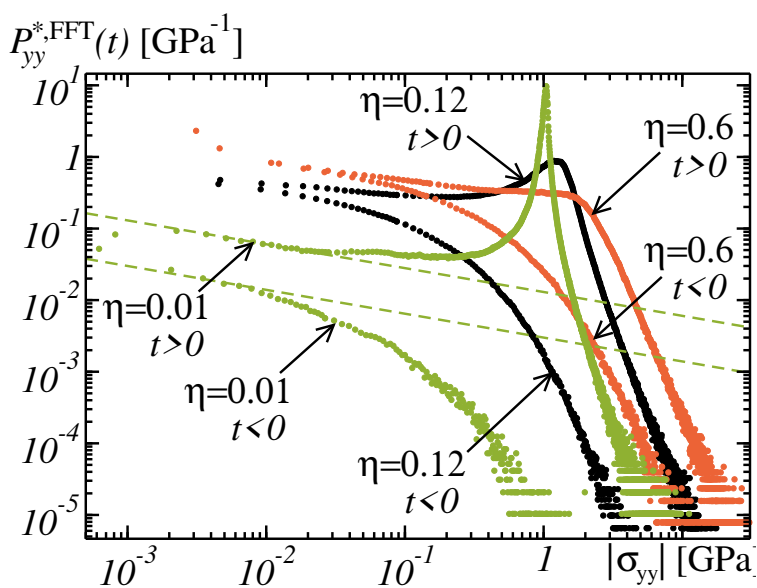

(a)

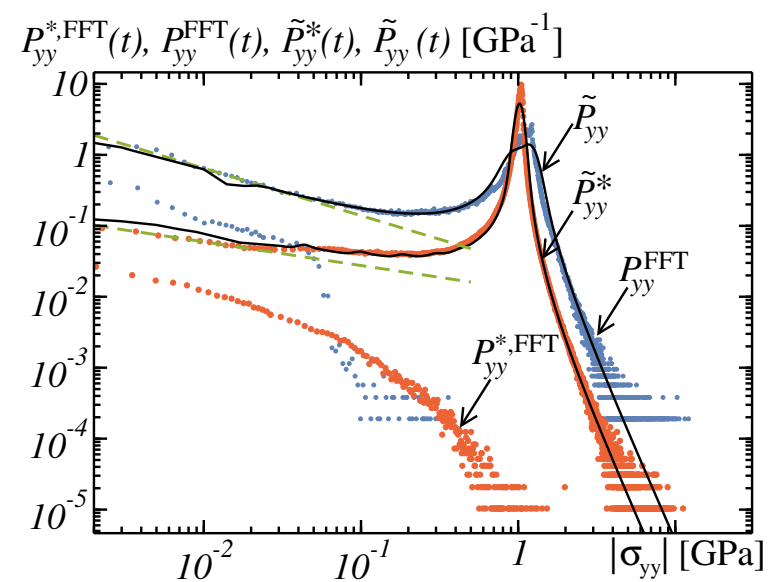

(b)

Figure 9. (a) Log-log plot of the probability distribution function $P_{y y}^{*, F F T}$ for the stress components $\sigma_{y y}$ in media with randomly-oriented cracks, as predicted by FFT computations. Dots: FFT data for $\eta=0.01,0.12$ and 0.6 . Dashed lines: powerlaws $\sim t^{-1 / 3}$ (see text). (b) Comparison between the probability distribution functions $P_{y y}^{F F T}$, $P_{y y}^{*, F F T}$, predicted by FFT (dots) and models $\widetilde{P}_{y y}$ and $\widetilde{P}_{y y}^{*}$ (solid lines), in log-log plot.

FFT data for the distribution $P_{x x, y y}^{*, F F T}$ are shown in Fig. (9) for varying values of the crack density parameter $\eta$. For $\eta=0.01$, the data is consistent with the prediction $\widetilde{P}_{y y}^{*}(t) \sim|t|^{-0.33}$ (Tab. 1) in the region $t \gtrsim 0$ (see dashed green lines with slope $-1 / 3$ ).

Fig. (9b) shows, in log-log scale, the distribution functions $P_{y y}^{F F T}$ and $P_{y y}^{*, F F T}$ for the stress component $\sigma_{y y}$ in media with parallel $(\eta=0.035)$ and randomly-oriented $(\eta=0.01)$ cracks, as computed by FFT (colored dots). This data is compared to the estimates $\widetilde{P}_{y y}$ and $\widetilde{P}_{y y}^{*}$ given by Eq. (31) (solid lines). The Van Hove powerlaw singularities for the distribution tails $(t \rightarrow \infty)$ and near zero $\left(t \rightarrow 0^{+}\right)$are accurately reproduced. Dashed green lines are powerlaw fits of the FFT data with exponent $-1 / 3$ and $-2 / 3$. When $t<0$ however, estimates $\widetilde{P}_{y y}$ and $\widetilde{P}_{y y}^{*}$ greatly underestimate the true distributions $P_{y y}^{F F T}(t)$ and $P_{y y}^{*, F F T}(t)$. Indeed, for negative values of $t$, the distributions $\widetilde{P}_{y y}$ and $\widetilde{P}_{y y}^{*}$ fall outside of the graph in Fig. (9b) and are not represented. But again, the present approach does not take into account mixed-mode loadings and all cracks are supposed open so that high negative values of the stress components are presumably induced by regions around crack tips subjected to compression or mixed-mode loadings, which are not taken into account in the present approach. 


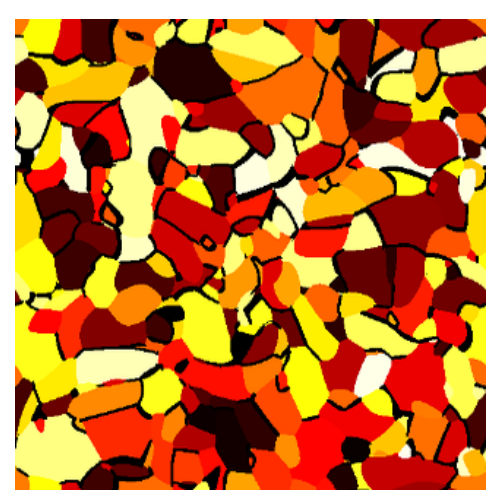

(a)

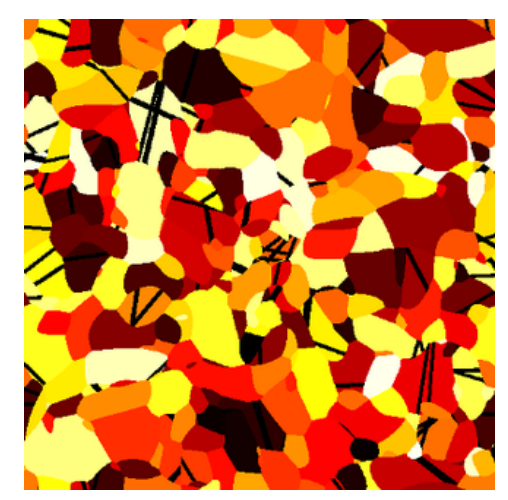

(b)

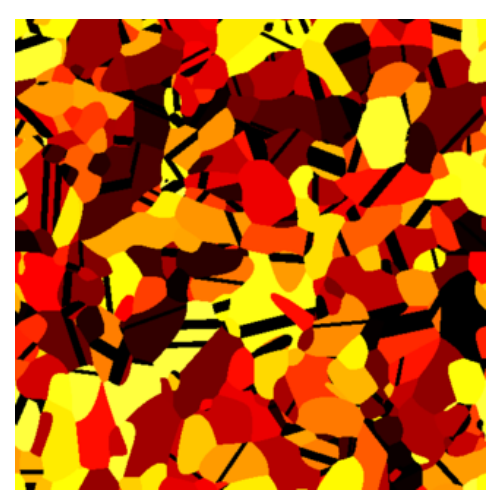

(c)

Figure 10. (a) Polycrystal model JM-I with integranular cracks. (b) Model JM-T with transgranular cracks. Cracks orientation uncorrelated to the crystal symmetry axis. (c) Model JM-TW with "weak-plane" transgranular cracks. Cracks oriented parallel to the basal plane in each crystal.

\section{Field distributions in cracked polycrystals}

This section is concerned with various models of cracked polycrystals based on the JohnsonMehl model and introduced in [19]. The first one is a polycrystal with intergranular cracks, denoted JM-I and represented in Fig. (10a). Three transgranular cracks models are also considered. In the first one, denoted (JM-T), crystals and cracks orientations are uncorrelated and uniformly-distributed on the sphere (Fig. 10b). In the other two transgranular crack models, the cracks orientation are correlated to the crystal directions. In the "weak-plane" transgranular model, denoted (JM-TW), cracks are oriented parallel to the crystal basal plane (Fig. 10c). In the stiff-plane model, denoted (JM-TS), the cracks are oriented perpendicular to the basal plane. Finally, for comparison purposes, we also consider a homogeneous body containing cracks, denoted (JM-IB), obtained by replacing the grains in model (JM-I) by a homogeneous isotropic medium with the same elastic properties as those of the sound polycrystal. The density of cracks is fixed to $\eta=0.24$ in all models.

The distributions of the equivalent strain $\varepsilon_{\mathrm{eq}}$, mean strain $\varepsilon_{\mathrm{m}}$ and shear strain $\varepsilon_{x y}$ are shown in Figs. (11a-f), for hydrostatic strain loading $\left\langle\varepsilon_{\mathrm{m}}\right\rangle=1$ and shear strain $\left\langle\varepsilon_{x y}\right\rangle=0.5$. Solid lines represent polycrystals with transgranular cracks. Dashed lines refer to the integranular model (JM-I) and homogeneous cracked medium (JM-IB). Overall, the field distributions differ significantly depending on the microstructure. In models with transgranular cracks, the correlation between the cracks orientation and the grains basal plane has a strong effect on the field distribution $\varepsilon_{\mathrm{m}}$, for hydrostatic strain loading (Figs. 11c).

All field distributions exhibit a singularity, or accumulation point at $\varepsilon=0$. For the von Mises equivalent deviatoric part $\varepsilon_{\text {eq }}$, a local peak appears near $\varepsilon=0$, in all models, whereas the distributions for $\varepsilon_{\text {eq }}$ and $\varepsilon_{x y}$ blow up at $\varepsilon=0$. The peak appears to be more important for model with intergranular cracks than for models with transgranular cracks.

The decay of the distribution tails for the strain component $\varepsilon_{x x}$ is shown in Fig. (12), for various models subjected to hydrostatic loading. The transgranular model (JM-T) and intergranular model (JM-I) as well as a sound polycrystal model, with Johnson-Mehl (JM) or Voronoi (V) tessellation are considered. Models (JM-T) and (JM-I) exhibit powerlaw decays $P_{\varepsilon_{x x}}(t) \sim t^{-\nu}$ as $t \rightarrow \infty$ with $\nu \approx 4.7 \approx 5$. This exponent is close to that obtained in plane strain (Sec. 3). As shown by Fig. (12), the distribution of the field $\varepsilon_{x x}$ decays at a much faster rate in sound polycrystal models (brown and orange curves) than in cracked polycrystal models (red and green curves). For Johnson-Mehl and Voronoi sound polycrystals, the rate of decay for the distribution $P_{\varepsilon_{x x}}(t)$ as $t \rightarrow \infty$ is close to that of a powerlaw. The exponent of the powerlaw is about -8 . It varies greatly with the realization of the model (not shown) but is nevertheless much lower than -5 . In any case, such powerlaw decay may result from the blow-up of the elastic fields at multimaterial corners, which has been studied, notably, in plane strain and 


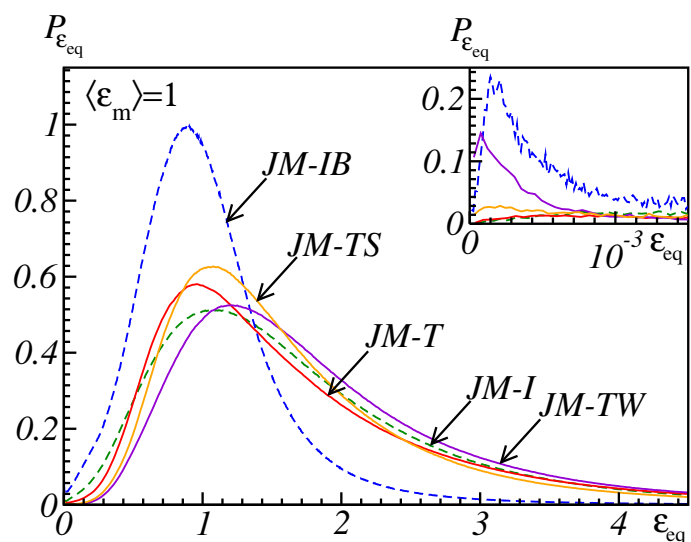

(a)

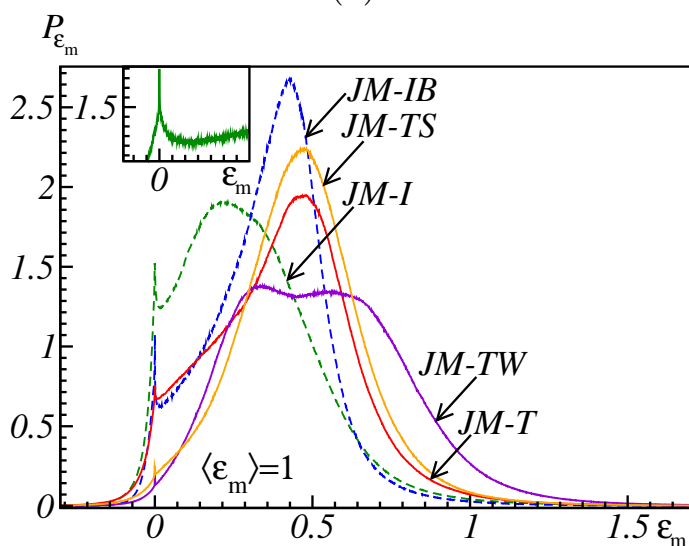

(c)

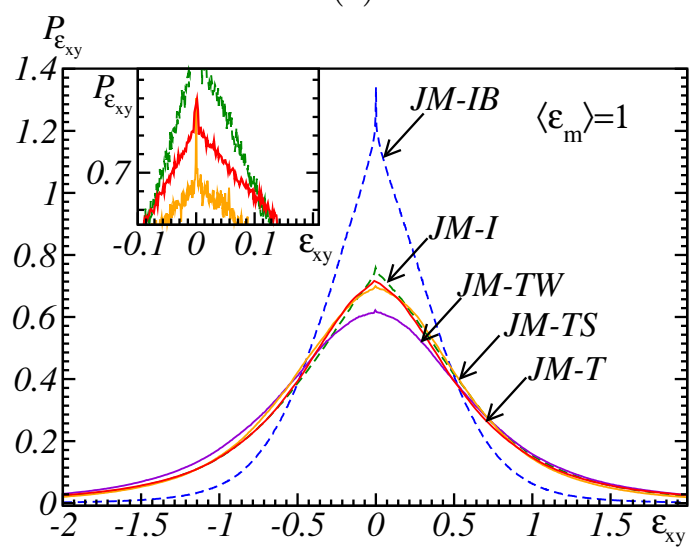

(e)

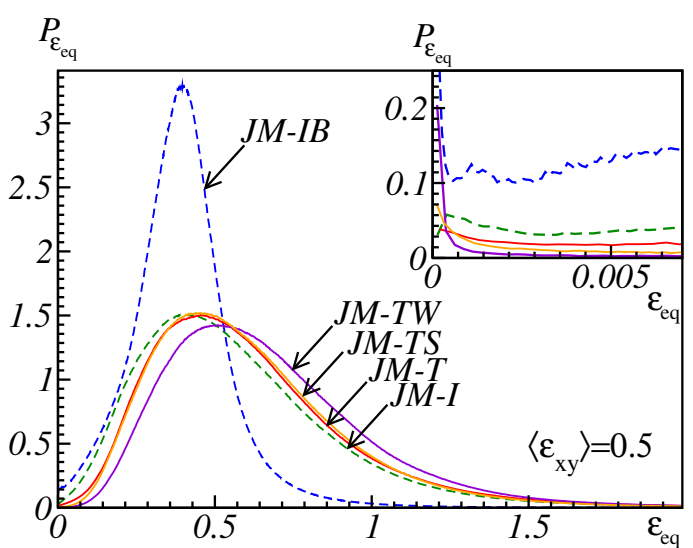

(b)

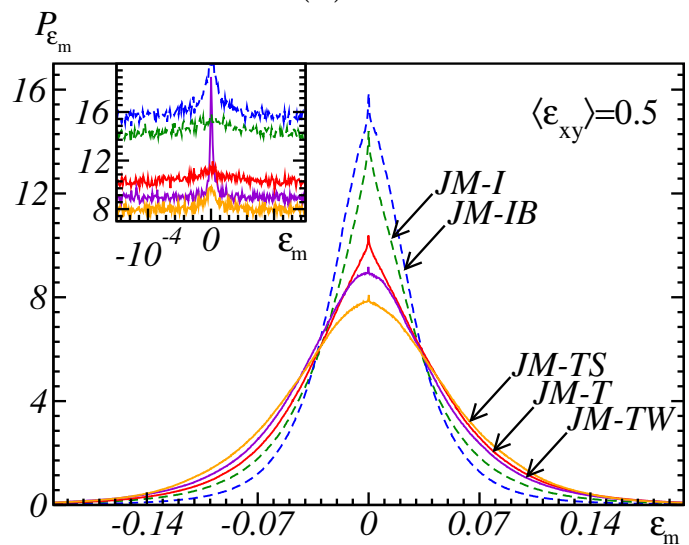

(d)

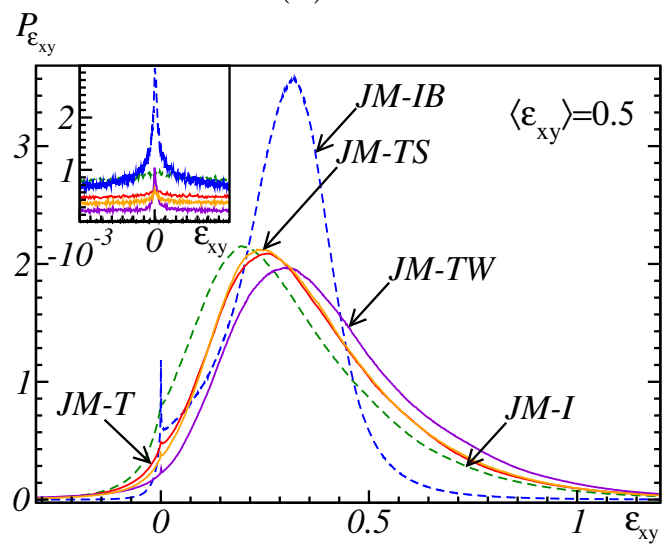

(f)

Figure 11. Histograms of the strain field components $\varepsilon_{\text {eq }}(\mathrm{a}, \mathrm{b}), \varepsilon_{\mathrm{m}}(\mathrm{c}, \mathrm{d}), \varepsilon_{x y}$ (e,f) in various cracked media, subjected to hydrostatic strain loading $(\mathrm{a}, \mathrm{c}, \mathrm{e})$ or shear (b,d,f). JM-I: Polycrystal with intergranular cracks. JM-T: transgranular cracks. JM-TW: "weak-plane" transgranular cracks. JM-TS: "stiff-plane" transgranular cracks. JM-IB: homogeneous cracked body. Embedded graphs: strain distribution in the region $\varepsilon \approx 0$. 


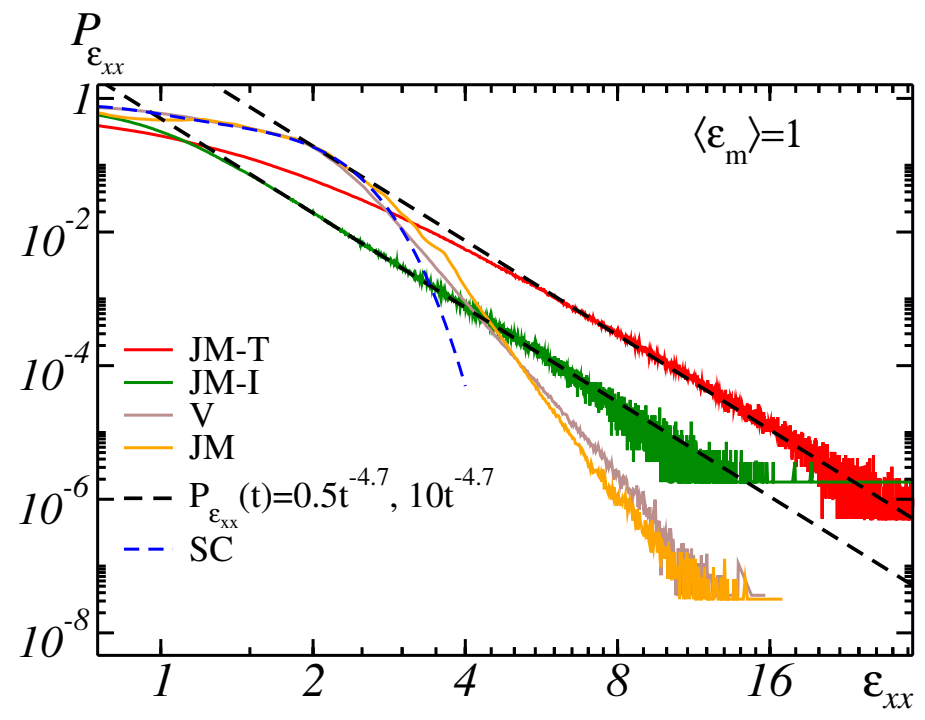

Figure 12. Tail of the distributions $P_{\varepsilon_{x x}}(t)(t \gg 1)$ of the strain field $\varepsilon_{x x}$ in media subjected to hydrostatic strain loading: comparison between sound Voronoi (V) and Johnson-Mehl (JM) polycrystals and cracked models JM-I (intergranular cracks) and JM-T (uncorrelated transgranular cracks), represented in log-log plot. Black dashed lines: powerlaw fits $P_{\varepsilon_{x x}}(t) \sim t^{-\nu}$ with $\nu=4.7$. SC: self-consistent estimate for a sound polycrystal.

plane stress problems $[5-7,31,32]$. The powerlaw decay is not predicted by the self-consistent method described in Sec. (2), which assumes a Gaussian intraphase field distribution (dashed blue line, Fig. 12).

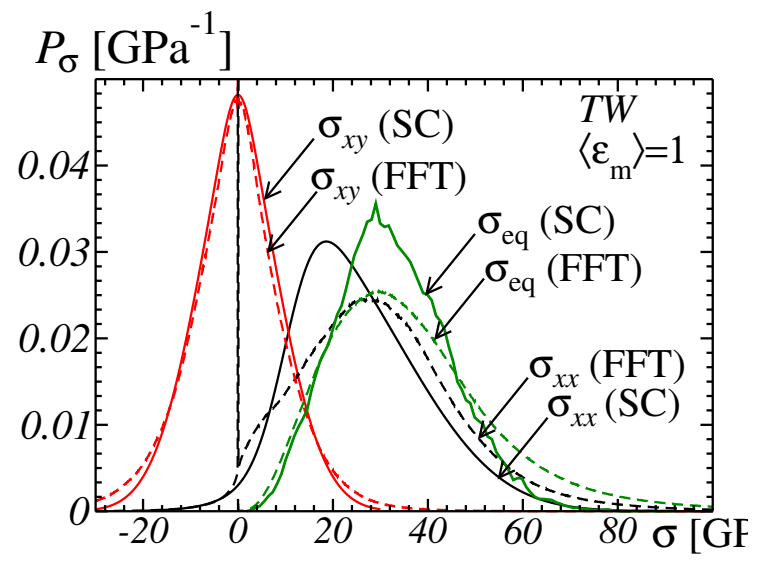

(a)

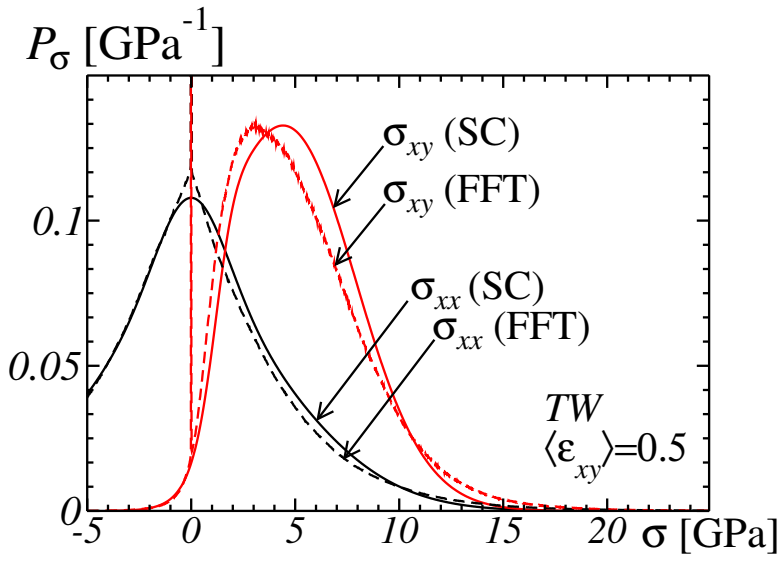

(b)

Figure 13. Distribution of stress components $\sigma_{x x}, \sigma_{\mathrm{eq}}$ and $\sigma_{x y}$ in a polycrystal with transgranular cracks oriented along basal planes (model "TW"). Solid lines: self-consistent estimates; dashed lines: FFT computations. (a): Hydrostatic strain loading. (b) Shear strain loading.

We close this section by further considerations regarding a particular model, the one containing weak-plane transgranular cracks (JM-TW). We make use of a self-consistent estimate, proposed in [19] for this model, which assumes separation of scales between the cracks and grains. In this method, the elastic moduli of each grain is weakened by the presence of microcracks. Thus, the same methodology as proposed in Sec. (2) may be applied to model (JM-TW), provided that the grains are replaced by weakened grains. Our results for the distribution of the stress components $\sigma_{\mathrm{eq}}, \sigma_{x x}$ and $\sigma_{x y}$ are shown in Fig. (13) and compared to FFT predictions. As previously, hydrostatic or shear loading is applied, and the FFT histograms are computed in the matrix only, as for the strain. In FFT computations, Van Hove singularities appear at $\boldsymbol{\sigma}=0$. As expected, the reconstruction making use of the self-consistent estimates are unable to reproduce such singularities. 


\section{Conclusion}

To investigate how one may use homogenization theories to predict the field probability distribution functions in linear elastic solids, the present contribution has examined two model problems in mechanics, that of a polycrystal and that of cracked media. Two different methods have been followed, based on an ansatz for the field distributions in grains or in regions surrounding a crack. Our main results are as follows.

The probability distribution function of the local elastic fields in sound polycrystals, with highly anisotropic grains and Johnson-Mehl or Voronoi tessellation microstructures, is accurately predicted by the self-consistent model and an hypothesis of multivariate Gaussian distribution of the intraphase strain and stress fields, except for the distribution tails. The method is not sufficient, however, when dealing with media containing cracks. The elastic fields in this case exhibit extended Van Hove singularities at $\varepsilon=0$ or $\boldsymbol{\sigma}=0$, even when the cracks are randomly-distributed and randomly-oriented.

For the model problem of a homogeneous body containing a population of cracks and subjected to plane strain, in particular, the probability distribution for the stress field exhibits a powerlaw decay $\sim\left|\sigma_{i j}\right|^{-5}$ as $\sigma_{i j} \rightarrow \pm \infty$, resulting from the singularity of the elastic field at the crack tip. A method has been proposed to reconstruct the probability distribution function of the stress fields, based on the assumption that the field surrounding each crack is governed by a stress intensity factor which follows a Gaussian probability distribution. The method gives accurate predictions for the probability distribution when the density of cracks is small, except for negative field values. Finally, numerical Fourier-based computations carried out in cracked media in $2 \mathrm{D}$ and $3 \mathrm{D}$ suggest a powerlaw decay $\sim\left|\sigma_{i j}\right|^{-5}$. In 3D cracked polycrystals, FFT data confirm the existence of Van Hove singularities at $\sigma_{i j}=0$.

Acknowledgments The financial support of the French Commissariat à l'Énergie Atomique is gratefully acknowledged. The authors thank J.-B. Gasnier for helping with the data in Fig. (11). F.W. thanks D. Leguillon for letting him know about references [31] and [32] and Y.-P. Pellegrini for helpful discussions.

\section{References}

[1] B. Abdallah, F. Willot, and D. Jeulin. Stokes flow through a Boolean model of spheres: Representative volume element. Transport in Porous Media, 109(3):711-726, 2015.

[2] AA Abrikosov, JC Campuzano, and K Gofron. Experimentally observed extended saddle point singularity in the energy spectrum of $\mathrm{YBa}_{2} \mathrm{Cu}_{3} \mathrm{O}_{6.9}$ and $\mathrm{YBa}_{2} \mathrm{Cu}_{4} \mathrm{O}_{8}$ and some of the consequences. Physica C: Superconductivity, 214(1-2):73-79, 1993.

[3] A. Ambos, F. Willot, D. Jeulin, and H. Trumel. Numerical modeling of the thermal expansion of an energetic material. International Journal of Solids and Structures, 60-61:125-139, 2015.

[4] T. W. Anderson. An introduction to multivariate statistical analysis, volume 2. Wiley, New York, 1958.

[5] A Barroso, V Mantič, and F París. Singularity analysis of anisotropic multimaterial corners. International Journal of Fracture, 119(1):1-23, 2003.

[6] A Barroso, V Mantič, and F París. Computing stress singularities in transversely isotropic multimaterial corners by means of explicit expressions of the orthonormalized Stroh-eigenvectors. Engineering Fracture Mechanics, 76(2):250-268, 2009.

[7] A. Barroso, D. Vicentini, V. Mantič, and F. París. Determination of generalized fracture toughness in composite multimaterial closed corners with two singular terms-part I: Test proposal and numerical analysis. Engineering Fracture Mechanics, 89:1-14, 2012.

[8] M. Barthélémy and H. Orland. Local field probability distribution in random media. Physical Review E, 56(3):2835, 1997.

[9] P. J. Basser and S. Pajevic. A normal distribution for tensor-valued random variables: applications to diffusion tensor mri. IEEE Transactions on Medical Imaging, 22(7):785-794, 2003.

[10] D. Bedrov, O. Borodin, G. D. Smith, T. D. Sewell, D. M. Dattelbaum, and L. L. Stevens. A molecular 
dynamics simulation study of crystalline 1,3,5-triamino-2,4,6-trinitrobenzene as a function of pressure and temperature. Journal of Chemical Physics, 131:224703, 2009.

[11] J. G. Berryman. Bounds and self-consistent estimates for elastic constants of random polycrystals with hexagonal, trigonal, and tetragonal symmetries. Journal of the Mechanics and Physics of Solids, 53(10):2141-2173, 2005.

[12] M. Bobeth and G. Diener. Field fluctuations in multicomponent mixtures. Journal of the Mechanics and Physics of Solids, 34:1-17, 1986.

[13] R. Brenner, O. Castelnau, and L. Badea. Mechanical field fluctuations in polycrystals estimated by homogenization techniques. Proceedings of the Royal Society of London, A460:3589-3612, 2004.

[14] R. Brenner, R.A. Lebensohn, and O. Castelnau. Elastic anisotropy and yield surface estimates of polycrystals. International Journal of Solids and Structures, 46:3018-3026, 2009.

[15] D. Cule and S. Torquato. Electric-field distribution in composite media. Physical Review B, 58(18):R11829, 1998.

[16] P.M. Duxbury, P.D. Beale, and C. Moukarzel. Breakdown of two-phase random resistor networks. Physical Review B, 51(6):3476, 1995.

[17] J.-B. Gasnier, F. Willot, H. Trumel, B. Figliuzzi, D. Jeulin, and M. Biessy. A Fourier-based numerical homogenization tool for an explosive material. Matériaux \& Techniques, 103(3):308, 2015.

[18] J.-B. Gasnier, F. Willot, H. Trumel, D. Jeulin, and J. Besson. Thermoelastic properties of microcracked polycrystals. Part I: Adequacy of Fourier-based methods for cracked elastic bodies. International Journal of Solids and Structures, 155:248-256, 2018.

[19] J.-B. Gasnier, F. Willot, H. Trumel, D. Jeulin, and M. Biessy. Thermoelastic properties of microcracked polycrystals. Part II: The case of jointed polycrystalline TATB. International Journal of Solids and Structures, 155:257-274, 2018.

[20] S. Giordano. Electrical behaviour of a single crack in a conductor and exponential laws for conductivity in micro cracked solids. International Journal of Applied Electromagnetics and Mechanics, 26(1, 2):119, 2007.

[21] S. Giordano and L. Colombo. Local elastic fields around cracks and their stress density of states. Physical Review B, 76(17):174120, 2007.

[22] H. Horii and S. Nemat-Nasser. Overall moduli of solids with microcracks: load-induced anisotropy. $J$. Mech. Phys. Solids, 31:155-171, 1983.

[23] M. I. Idiart, H. Moulinec, P. Ponte Castañeda, and P. Suquet. Macroscopic behavior and field fluctuations in viscoplastic composites: second-order estimates versus full-field simulations. Journal of the Mechanics and Physics of Solids, 54(5):1029-1063, 2006.

[24] V. John, I. Angelov, A. A. Öncül, and D. Thévenin. Techniques for the reconstruction of a distribution from a finite number of its moments. Chemical Engineering Science, 62(11):2890-2904, 2007.

[25] M. E. Johnson. Multivariate statistical simulation: A guide to selecting and generating continuous multivariate distributions. John Wiley \& Sons, New York, 2013.

[26] N. Kowalski, L. Delannay, P. Yan, and J.-F. Remacle. Finite element modeling of periodic polycrystalline aggregates with intergranular cracks. International Journal of Solids and Structures, 90:60-68, 2016.

[27] W. Kreher. Residual stresses and stored elastic energy of composites and polycrystals. Journal of the Mechanics and Physics of Solids, 38(1):115-128, 1990.

[28] VI Kushch, SV Shmegera, and I Sevostianov. Sif statistics in micro cracked solid: effect of crack density, orientation and clustering. International Journal of Engineering Science, 47(2):192-208, 2009.

[29] R.A. Lebensohn, O. Castelnau, R. Brenner, and P. Gilormini. Study of the antiplane deformation of linear 2D polycrystals with different microstructure. International Journal of Solids and Structures, 42:5441-5459, 2005.

[30] R.A. Lebensohn, Y. Liu, and P. Ponte Castañeda. On the accuracy of the self-consistent approximation for polycrystals: comparison with full-field numerical simulations. Acta Materialia, 52:5347-5361, 2004 .

[31] V. Mantič, A. Barroso, and F. París. Singular elastic solutions in anisotropic multimaterial corners: applications to composites. In V. Mantič, editor, Mathematical methods and models in composites, pages 425-495. Imperial College Press, London, 2014.

[32] V. Mantič, F. Paris, and J. Canas. Stress singularities in 2D orthotropic corners. International Journal of Fracture, 83(1):67-90, 1997.

[33] J.L. Meijering. Interface area, edge length, and number of vertices in crystal aggregates with random nucleation. Philips Res. Rep., 8:270-290, 1953. 
[34] P. Meunier and E. Villermaux. Van Hove singularities in probability density functions of scalars. Comptes Rendus Mécanique, 335(3):162-167, 2007.

[35] R.E. Miles. The random division of space. Advances in applied probability, pages 243-266, 1972.

[36] H. Moulinec and P. Suquet. A fast numerical method for computing the linear and non linear mechanical properties of the composites. Comptes Rendus de l'Académie des Sciences Série II, 318:1417-1423, 1994.

[37] Y.-P. Pellegrini. Field distributions and effective-medium approximation for weakly nonlinear media. Physical Review B, 61(14):9365, 2000.

[38] P. Ponte Castañeda and P. Suquet. Nonlinear composites. In Advances in applied mechanics, volume 34, pages 171-302. Elsevier, 1998.

[39] D. Ruer. Méthode vectorielle d'analyse de la texture. PhD thesis, Université de Metz, France, 1976.

[40] J. Serra. The boolean model and random sets. Computer Graphics and Image Processing, 12(2):99$126,1980$.

[41] I. Sevostianov and V. Kushch. Effect of pore distribution on the statistics of peak stress and overall properties of porous material. International Journal of Solids and Structures, 46(25-26):4419-4429, 2009.

[42] J. A. Shohat and J. D. Tamarkin. The problem of moments, volume 1. American Mathematical Soc., Providence, 1943.

[43] C.T. Sun and Z.H. Jin. Fracture Mechanics, volume 10. Academic Press, Waltham, 2012.

[44] P. Suquet and H. Moulinec. Mathematics of multiscale materials. In K.M. Golden, G.R. Grimmett, R.D. James, G.W. Milton, and P.N. Sen, editors, IMA Lecture Notes 99, chapter Numerical simulation of the effective elastic properties of a class of cell materials. Springer-Verlag, New York, 1998.

[45] L. Van Hove. The occurrence of singularities in the elastic frequency distribution of a crystal. Physical Review, 89(6):1189, 1953.

[46] M. L. Williams. On the stress distribution at the base of a stationary crack. Journal of Applied Mechanics, 24:109-114, 1957.

[47] J.R. Willis. Bounds and self-consistent estimates for the overall properties of anisotropic composites. Journal of the Mechanics and Physics of Solids, 25:185-202, 1977.

[48] F. Willot. Fourier-based schemes for computing the mechanical response of composites with accurate local fields. Comptes Rendus Mécanique, 343(3):232-245, 2015.

[49] F. Willot and Y.-P. Pellegrini. Fast Fourier transform computations and build-up of plastic deformation in 2D, elastic-perfectly plastic, pixelwise-disordered porous media. In D. Jeulin, S. Forest (eds), "Continuum Models and Discrete Systems CMDS 11", pages 443-449, Paris, 2008. École des Mines. Online at https://arxiv.org/abs/0802.2488.

[50] F. Willot, Y.-P. Pellegrini, and P. Ponte Castañeda. Localization of elastic deformation in strongly anisotropic, porous, linear materials with periodic microstructures: exact solutions and dilute expansions. Journal of the Mechanics and Physics of Solids, 56(4):1245-1268, 2008. 